\title{
Local abelian Kato-Parshin reciprocity law: A survey
}

\author{
Kâzım İlhan İkeda*1,2 (iD, Erol Serbest ${ }^{3}$ iD \\ ${ }^{1}$ Department of Mathematics, Boğaziçi University, 34342 Bebek, İstanbul, Turkey \\ ${ }^{2}$ Feza Gürsey Center for Physics and Mathematics, Boğaziçi University-Kandilli Campus, Rasathane \\ Cad., Kandilli Mah., 34684, İstanbul, Turkey \\ ${ }^{3}$ Department of Mathematics, Yeditepe University, İönü Mah. Kayışdağı Cad. 326A, 26 Ağustos \\ Yerleşimi, 34755 Ataşehir, İstanbul, Turkey
}

-In memory of Seydin Serbest-

\begin{abstract}
Let $K$ denote an $n$-dimensional local field. The aim of this expository paper is to survey the basic arithmetic theory of the $n$-dimensional local field $K$ together with its Milnor $K$ theory and Parshin topological $K$-theory; to review Kato's ramification theory for finite abelian extensions of the $n$-dimensional local field $K$, and to state the local abelian higherdimensional $K$-theoretic generalization of local abelian class field theory of Hasse, which is developed by Kato and Parshin. The paper is geared toward non-abelian generalization of this theory.
\end{abstract}

Mathematics Subject Classification (2020). 11S70, 19F05

Keywords. higher-dimensional local fields, Milnor $K$-theory, local class field theory

\section{Introduction}

The aim of this paper is to survey the local abelian higher-dimensional $K$-theoretic generalization of the local abelian class field theory of Hasse [16] developed by Parshin (in positive characteristic) $[42,44,45]$ and by Kato (in general) $[22,23,25]$ in the late $1970 \mathrm{~s}$ and early 80's; namely, the local abelian Kato-Parshin class field theory, which has later been simplified, made explicit, and cohomology free by Fesenko [7-9].

For a field $K$, let $K^{\mathrm{ab}}$ denote the maximal abelian extension of $K$ in a fixed separable closure $K^{\mathrm{sep}}$ of $K$. Then the maximal abelian Hausdorff quotient $G_{K}^{\mathrm{ab}}$ of the absolute Galois group $G_{K}=\operatorname{Gal}\left(K^{\mathrm{sep}} / K\right)$ is naturally isomorphic to $\operatorname{Gal}\left(K^{\mathrm{ab}} / K\right)$. In particular, if $K$ is a non-archimedean local field; i.e., a complete discrete valuation field with finite residueclass field $\kappa_{K}=O_{K} / \mathfrak{p}_{K}$ of $q=p^{f}$ elements, where $O_{K}$ denotes the ring of integers of $K$, $\mathfrak{p}_{K}$ its unique maximal ideal, and $p$ a prime number; that is, if $K$ is either a finite extension of $\mathbb{Q}_{p}$ in case $\operatorname{char}(K)=0$, or a finite extension of $\mathbb{F}_{p}((X))$ in case $\operatorname{char}(K)=p>0$, then

\footnotetext{
*Corresponding Author.

Email addresses: kazimilhan.ikeda@boun.edu.tr (K. I. Ikeda), erol.serbest@yeditepe.edu.tr (E. Serbest)

Received: 01.12.2020; Accepted: 27.03.2021
} 
$\operatorname{Gal}\left(K^{\mathrm{ab}} / K\right)$ and the profinite completion $\widehat{K^{\times}}$of the multiplicative group $K^{\times}$of the nonarchimedean local field $K$ are both algebraically and topologically isomorphic via local abelian Hasse reciprocity law

$$
\operatorname{Rec}_{K}: \widehat{K^{\times}} \stackrel{\sim}{\rightarrow} \operatorname{Gal}\left(K^{\mathrm{ab}} / K\right)
$$

of $K$. This isomorphism has many salient features. For instance, via this arrow, $\widehat{K^{x}}$ encodes all of the arithmetic information on the abelian extensions of the non-archimedean local field $K$, which is the subject matter of local abelian class field theory of $K$. A detailed exposition of local fields and local abelian class field theory in modern terms can be found in $[15,21]$.

Now, let $F$ be a global field; that is, $F$ is either a finite extension of $\mathbb{Q}$ in case $\operatorname{char}(F)=$ 0 , or a finite extension of $\mathbb{F}_{p}(X)$ in case $\operatorname{char}(F)=p>0$. The completion $F_{\nu}$ of $F$ with respect to a finite place $\nu$ of $F$ is a non-archimedean local field. Following the "idèlic philosophy" of Chevalley, global class field theory of $F$ can be constructed by glueing the local abelian class field theories of $F_{\nu}$ for all $\nu$ [2]. In recent years however, the arithmetic study of global fields extended its scope and instead of considering only global fields; that is, integral schemes $X$ of absolute dimension 1, higher-dimensional integral schemes $X$ are taken into consideration. In this setting, let $F$ denote the field of rational functions on an integral scheme $X$ of absolute dimension $n$. Then to any flag of irreducible non-singular subschemes $X_{0} \subset X_{1} \subset \cdots \subset X_{n}=X$ of $X$ with $\operatorname{dim}\left(X_{i}\right)=i$ for $i=0,1, \cdots, n$, Parshin introduced a completion $F_{\left(X_{0}, \cdots, X_{n}\right)}$ of $F$, which is an example of an $n$-dimensional local field. Recall that, an $n$-dimensional local field has an inductive definition: for $n \geq 1$, an $n$-dimensional local field is a complete discrete valuation field whose residue field is an $(n-1)$-dimensional local field, where in this terminology 0-dimensional local fields are finite fields and 1-dimensional local fields are the "classical" non-archimedean local fields. The collection of such $n$-dimensional local fields $F_{\left(X_{0}, \cdots, X_{n}\right)}$ over all possible flags $\left(X_{0}, \cdots, X_{n}\right)$ of the scheme $X$ plays a central role in the global class field theory of the scheme $X$, a grand theory again created by Parshin $^{\dagger}$, Bloch $^{\ddagger}$, Kato and S. Saito ${ }^{\S}$, which is constructed, following the "higher-dimensional idèlic philosophy" of Beilinson and Parshin [18], by glueing the local abelian $n$-dimensional class field theories of $F_{\left(X_{0}, \cdots, X_{n}\right)}$ for all $\left(X_{0}, \cdots, X_{n}\right)$.

The aim of this work is to survey the local abelian $n$-dimensional class field theory; namely, the study of arithmetic information on the abelian extensions of an $n$-dimensional local field $K$ encoded in the local abelian $n$-dimensional reciprocity law

$$
\operatorname{Rec}_{K}: \widehat{\mathrm{K}}_{n}^{\mathrm{top}}(K) \stackrel{\sim}{\rightarrow} \operatorname{Gal}\left(K^{\mathrm{ab}} / K\right)
$$

of the $n$-dimensional local field $K$, where $\widehat{\mathrm{K}}_{n}^{\text {top }}(K)$ is the profinite completion of the $n$-th Parshin topological $K$-group $\mathrm{K}_{n}^{\text {top }}(K)$ of $K$, which is an algebraic, analytic and topological object depending only and solely to the ground field $K$. Moreover, in the particular case $n=1$, this arrow reduces to the ordinary local abelian Hasse reciprocity law of $K$. In the local abelian $n$-dimensional theory:

- Non-archimedean local fields $K$ are replaced by $n$-dimensional local fields $K$ non-archimedean local fields $K \rightsquigarrow n$-dimensional local fields $K$,

\footnotetext{
${ }^{\dagger}$ Parshin developed the global class field theory of algebraic surfaces using his 2-dimensional adèles [43,44]. ${ }^{\ddagger}$ Bloch is one of the first researchers who used algebraic $K$-theory to construct the class field theory of arithmetic surfaces [4].

$\S$ Kato and S. Saito studied the global class field theory of arithmetic surfaces and then extended their results to arbitrary dimensional arithmetic schemes [26,27].
} 
- and multiplicative groups $K^{\times}$of non-archimedean local fields $K$ are replaced by the $n$-th Parshin topological $K$-groups $\mathrm{K}_{n}^{\text {top }}(K)$ of $n$-dimensional local fields $K$

$$
\text { the group } \widehat{K^{\times}} \rightsquigarrow \text { the group } \widehat{\mathrm{K}}_{n}^{\mathrm{top}}(K) \text {, }
$$

hence the name " $K$-theoretic generalization" of local abelian class field theory, or the local abelian Kato-Parshin class field theory.

The paper is organised as follows.In Sections 2 and 3, we shall respectively review the basic arithmetical theory and the topological theory of $n$-dimensional local fields. Next, in Sections 4 and 5, Milnor $K$-theory and Parshin topological $K$-theory of $n$-dimensional local fields are discussed. In Section 6, after reviewing ramification theory for non-archimedean local fields, we sketch Kato's ramification theory, which is defined only for abelian extensions of $n$-dimensional local fields introduced in [24,28], and note that Kato's ramification theory for finite abelian extensions of $n$-dimensional local fields is compatible with the local abelian Kato-Parshin reciprocity law. Finally, in Section 7, we state the local abelian $K$-theoretic class field theory of Kato and Parshin. In this section, we stick to the methods introduced by Fesenko, as his methods have advantages for the non-abelian generalization of this theory [20].

\section{2. n-dimensional local fields}

The main references for this section are [33] and the excellent reviews [36, 37, 41, 50]. Let $K$ be an $n$-dimensional local field. That is, attached to $K$, there exists a sequence of fields

$$
K_{0}, K_{1}, \cdots, K_{n-1}, K_{n}=K,
$$

called the Parshin chain of $K$, where

$-K_{i+1}$ is a complete discrete valuation field endowed with a discrete valuation

$$
\nu_{K_{i+1}}: K_{i+1} \rightarrow \mathbb{Z} \cup\{\infty\}
$$

with the ring of integers $O_{\nu_{K_{i+1}}}=O_{K_{i+1}}$ having the unique maximal ideal $\mathfrak{p}_{\nu_{K_{i+1}}}=$ $\mathfrak{p}_{K_{i+1}}$ for every $i=0, \cdots, n-1$;

- The residue-class field $\kappa_{\nu_{K_{i+1}}}=\kappa_{K_{i+1}}$ of $K_{i+1}$ is $K_{i}$ for every $i=0, \cdots, n-1$;

- $K_{0}=\mathbb{F}_{q}$ the finite field with $q=p^{s}$ elements, where $p$ denotes a prime number (we could have assumed $K_{0}$ is a perfect field instead).

The residue-class field $K_{n-1}$ of $K_{n}$ is called the first residue-class field of the $n$-dimensional local field $K$, and the residue-class field $K_{0}=\mathbb{F}_{q}$ of $K_{1}$ is called the last residue-class field of the $n$-dimensional local field $K$. Moreover, $K$ is said to be a mixed-characteristic $n$-dimensional local field if $\operatorname{char}(K)=0$ and $\operatorname{char}\left(K_{n-1}\right)=p>0$, and called an equalcharacteristic $n$-dimensional local field if $\operatorname{char}(K)=\operatorname{char}\left(K_{n-1}\right)$.

Here are some examples of $n$-dimensional local fields:

Example 2.1. Observe that,

$$
K=L\left(\left(X_{1}\right)\right) \cdots\left(\left(X_{n-1}\right)\right)
$$

where $L$ is a non-archimedean local field, is a natural example of an $n$-dimensional local field.

\footnotetext{
\Note that, Kato's ramification theory introduced in [24] is for abelian extensions of $n$-dimensional local fields, while Abbes and T. Saito's ramification theory [1] is for general Galois extensions of $n$-dimensional local fields [49]. On the other hand, Abbes-Saito ramification theory for abelian extensions of $n$-dimensional local fields coincides with Kato's ramification filtration [28].
} 
Example 2.2. Let $k$ be a complete discrete valuation field with respect to a discrete valuation $\nu_{k}: k \rightarrow \mathbb{Z} \cup\{\infty\}$. The field

$$
K=k\{\{X\}\}=\left\{\sum_{i=-\infty}^{+\infty} c_{i} X^{i} \mid c_{i} \in k, \inf \left\{\nu_{k}\left(c_{i}\right) \mid i \in \mathbb{Z}\right\}>-\infty, \lim _{i \rightarrow-\infty} \nu_{k}\left(c_{i}\right)=+\infty\right\}
$$

endowed with a discrete valuation

$$
\nu_{K}: K \rightarrow \mathbb{Z} \cup\{\infty\}
$$

defined by

$$
\nu_{K}\left(\sum_{i=-\infty}^{+\infty} c_{i} X^{i}\right)=\inf \left\{\nu_{k}\left(c_{i}\right) \mid i \in \mathbb{Z}\right\}
$$

for every $\sum_{i=-\infty}^{+\infty} c_{i} X^{i} \in K$, is a complete discrete valuation field with residue class field $\kappa_{K}=\kappa_{k}((X))$.

Therefore, for a non-archimedean local field $L$, and for $0 \leq j \leq n-1$,

$$
K=L\left\{\left\{X_{1}\right\}\right\} \cdots\left\{\left\{X_{j}\right\}\right\}\left(\left(X_{j+2}\right)\right) \cdots\left(\left(X_{n}\right)\right)
$$

is an $n$-dimensional local field, called a standard $n$-dimensional local field, following [36, 50]. The extreme cases $j=0$ and $j=n-1$ mean $K=L\left(\left(X_{2}\right)\right) \cdots\left(\left(X_{n}\right)\right)$ and $K=$ $L\left\{\left\{X_{1}\right\}\right\} \cdots\left\{\left\{X_{n-1}\right\}\right\}$, respectively.

Remark 2.3. Let $k$ be a complete discrete valuation field with respect to a discrete valuation $\nu_{k}: k \rightarrow \mathbb{Z} \cup\{\infty\}$. Then, $k\left(\left(X_{1}\right)\right)\left\{\left\{X_{2}\right\}\right\}$ is isomorphic to $k\left(\left(X_{2}\right)\right)\left(\left(X_{1}\right)\right)$. So, it suffices to consider standard higher-dimensional local fields. For details, look at the classification theorem for $n$-dimensional local fields that we recall below.

Assumption 2.4. From now on, all through the paper, $K$ denotes an $n$-dimensional local field with the corresponding Parshin chain

$$
\mathbb{F}_{q}=K_{0}, K_{1}, \cdots, K_{n-1}, K_{n}=K \text {. }
$$

Notation 2.5. To simplify the discussion, for $a \in O_{K_{n}}$ and for an integer $i$ satisfying $0 \leq i \leq n-1$, let $\bar{a}^{(n, \cdots, n-i)}$ denote the element in $K_{n-i-1}$ defined by "succesive reductions of $a$ modulo maximal ideals $\mathfrak{p}_{K_{n}}, \cdots, \mathfrak{p}_{K_{n-i}}$ respectively" as

$$
a \quad\left(\bmod \mathfrak{p}_{K_{n}}\right) \quad\left(\bmod \mathfrak{p}_{K_{n-1}}\right) \cdots \quad\left(\bmod \mathfrak{p}_{K_{n-i}}\right)
$$

provided that $\bar{a}^{(n)} \in O_{K_{n-1}}, \bar{a}^{(n, n-1)} \in O_{K_{n-2}}, \cdots, \bar{a}^{(n, \cdots, n-i+1)} \in O_{K_{n-i}}$. Note that, $\bar{a}^{(n, \cdots, n-i)}$ is a non-zero element of $K_{n-i-1}$ if $\bar{a}^{(n)} \in O_{K_{n-1}}^{\times}=U_{K_{n-1}}, \bar{a}^{(n, n-1)} \in O_{K_{n-2}}^{\times}=$ $U_{K_{n-2}}, \cdots, \bar{a}^{(n, \cdots, n-i+1)} \in O_{K_{n-i}}^{\times}=U_{K_{n-i}}$.

An $n$-tuple $\Pi_{K}=\left(t_{1, K}, \cdots, t_{n, K}\right)$ in $K^{n}$ is called a system of local parameters of $K$, if

(1) $t_{n, K}$ is a prime element of $K_{n}$ with respect to $\nu_{K_{n}}$;

(2) $t_{n-1, K} \in U_{K_{n}}$ and its residue class $\bar{t}_{n-1, K}^{(n)}:=t_{n-1, K}\left(\bmod \mathfrak{p}_{K_{n}}\right)$ modulo $\mathfrak{p}_{K_{n}}$ is a prime element of $K_{n-1}$ with respect to $\nu_{K_{n-1}}$;

(n) $t_{1, K} \in U_{K_{n}}$ such that $\bar{t}_{1, K}^{(n)} \in U_{K_{n-1}}, \cdots, \bar{t}_{1, K}^{(n, \cdots, 3)} \in U_{K_{2}}$ and $\bar{t}_{1, K}^{(n, \cdots, 2)}$ is a prime element of $K_{1}$ with respect to $\nu_{K_{1}}$.

So, following [33] and [42,44,45], $n$-dimensional local fields can be classified as follows. For the $n$-dimensional local field $K$ :

- If $\operatorname{char}(K)=p$, then it is possible to choose $t_{1}, \cdots, t_{n} \in K$, such that

$$
K \stackrel{\sim}{\rightarrow} \mathbb{F}_{q}\left(\left(t_{1}\right)\right) \cdots\left(\left(t_{n}\right)\right) .
$$

Moreover, $\left(t_{1}, \cdots, t_{n}\right) \in K^{n}$ becomes a system of local parameters of $K$; 
- If $\operatorname{char}\left(K_{1}\right)=0$, then it is possible to choose $t_{2}, \cdots, t_{n} \in K$, such that

$$
K \stackrel{\sim}{\rightarrow} K_{1}\left(\left(t_{2}\right)\right) \cdots\left(\left(t_{n}\right)\right) .
$$

Moreover, choosing $\pi_{1, K} \in U_{K_{n}}$ such that $\bar{\pi}_{1, K}^{(n)} \in U_{K_{n-1}}, \cdots, \bar{\pi}_{1, K}^{(n, \cdots, 3)} \in U_{K_{2}}$ and $\bar{\pi}_{1, K}^{(n, \cdots, 2)}$ is a prime element $\pi_{K_{1}}$ of $K_{1}$ with respect to $\nu_{K_{1}}$, the $n$-tuple $\left(\pi_{K_{1}}, t_{2}, \cdots, t_{n}\right) \in K^{n}$ becomes a system of local parameters of $K$;

- If none of the above holds, there exists a unique $r \in\{1, \cdots, n-1\}$ such that $\operatorname{char}\left(K_{r+1}\right) \neq \operatorname{char}\left(K_{r}\right)$. Then, there exists a unique non-archimedean local field $L$ of char. 0 , and there exist $n-1$ elements $t_{1}, \cdots, t_{r}, t_{r+2}, \cdots, t_{n} \in K$, such that $K$ is a finite extension of the standard field

$$
L\left\{\left\{t_{1}\right\}\right\} \cdots\left\{\left\{t_{r}\right\}\right\}\left(\left(t_{r+2}\right)\right) \cdots\left(\left(t_{n}\right)\right) .
$$

Moreover, if $\operatorname{char}\left(K_{0}\right)=p$, then $L$ may be chosen to be the unique unramified extension of $\mathbb{Q}_{p}$ with residue-class field $K_{0}$.

Now, fix a system of local parameters $\Pi_{K}=\left(t_{1, K}, \cdots, t_{n, K}\right) \in K^{n}$ of $K$. This system of local parameters $\Pi_{K}$ of $K$ naturally determines a mapping

$$
\rho_{\Pi_{K}}: K \rightarrow K_{1} \times \cdots \times K_{n}
$$

defined by

$$
\rho_{\Pi_{K}}: a \mapsto\left(a_{1}, \cdots, a_{n}\right),
$$

where $a_{n}=a \in K_{n}$ and $a_{i}=\bar{a}_{i+1}^{(i+1)}\left(\bar{t}_{i+1, K}^{(n, \cdots, i+1)}\right)^{-\nu_{K_{i+1}}\left(a_{i+1}\right)} \in K_{i}$ for $1 \leq i \leq n-1$. Then, there exists a rank $n$ discrete valuation

$$
\bar{v}_{K}=\left(\nu_{K_{1}}, \cdots, \nu_{K_{n}}\right) \circ \rho_{\Pi_{K}}: K \stackrel{\rho_{\Pi_{K}}}{\longrightarrow} K_{1} \times \cdots \times K_{n} \stackrel{\left(\nu_{K_{1}}, \cdots, \nu_{K_{n}}\right)}{\longrightarrow} \mathbb{Z}^{n} \cup\{\infty\}
$$

on $K$ defined by

$$
\bar{v}_{K}(a):=\left(\nu_{K_{1}}, \cdots, \nu_{K_{n}}\right) \circ \rho_{\Pi_{K}}(a)=\left(\nu_{K_{1}}\left(a_{1}\right), \cdots, \nu_{K_{n}}\left(a_{n}\right)\right)
$$

for $a \in K^{\times}$. Here, $\mathbb{Z}^{n}$ is assumed to be lexicographically ordered in the sense of Madunts and Zhukov as follows: For $\boldsymbol{i}=\left(i_{1}, \cdots, i_{n}\right), \boldsymbol{j}=\left(j_{1}, \cdots, j_{n}\right) \in \mathbb{Z}^{n}$,

$$
\boldsymbol{i} \prec \boldsymbol{j} \Longleftrightarrow i_{\ell}<j_{\ell}, i_{\ell+1}=j_{\ell+1}, \cdots, i_{n}=j_{n} \text { for some } 0 \leq \ell \leq n .
$$

Recall that, this rank $n$ discrete valuation $\bar{v}_{K}$ on $K$ depends on the system of local parameters $\Pi_{K}$ of $K$. However, if $\Pi_{K}^{\prime} \in K^{n}$ is another system of local parameters of $K$, then the corresponding rank $n$ discrete valuation $\bar{v}_{K}^{\prime}$ on $K$ is equivalent to $\bar{v}_{K}$ in the following sense:

$$
\bar{v}_{K}^{\prime}(a)=\bar{v}_{K}(a) T,{ }^{\forall} a \in K,
$$

where $T=\left(v_{K_{j}}^{\prime}\left(\operatorname{Proj}_{j} \circ \rho_{\Pi_{K}}\left(t_{i, K}\right)\right)\right)_{1 \leq i, j \leq n} \in \mathrm{M}(n, \mathbb{Z})$, which is a lower triangular square integral matrix of size $n$ with the unit element 1 on the main diagonal. Here, $\operatorname{Proj}_{j}$ : $K_{1} \times \cdots \times K_{n} \rightarrow K_{j}$ denotes the projection map on the $j^{\text {th }}$ coordinate. As usual, $\mathrm{M}(n, \mathbb{Z})$ denotes the set of all integral square matrices of size $n$. The rank $n$ discrete valuation $\bar{v}_{K}$ on $K$ is called normalized, if $\bar{v}_{K}\left(K^{\times}\right)=\mathbb{Z}^{n}$.

For a rank $n$ discrete valuation $\bar{v}_{K}: K \rightarrow \mathbb{Z}^{n} \cup\{\infty\}$ defined on $K$, introduce the subring $O_{\bar{v}_{K}}$ of $K$ by

$$
O_{\bar{v}_{K}}=\left\{a \in K \mid \bar{v}_{K}(a) \succeq \mathbf{0}\right\},
$$

where $\mathbf{0}=(0, \cdots, 0) \in \mathbb{Z}^{n}$, which is called the ring of integers of $K$ with respect to the rank $n$ discrete valuation $\bar{v}_{K}$. Note that $O_{\bar{v}_{K}}$ has the unique maximal ideal $\mathfrak{p}_{\bar{v}_{K}}$ defined by

$$
\mathfrak{p}_{\bar{v}_{K}}=\left\{a \in O_{\bar{v}_{K}} \mid \bar{v}_{K}(a) \succ \mathbf{0}\right\} .
$$

The quotient field $O_{\bar{v}_{K}} / \mathfrak{p}_{\bar{v}_{K}}=: \kappa_{\bar{v}_{K}}$, called the residue class field of $K$ with respect to the rank $n$ discrete valuation $\bar{v}_{K}$, is isomorphic to $K_{0}=\mathbb{F}_{q}$. 
The arithmetic structure of $O_{\bar{v}_{K}}$ has the following description. Introduce for each $\ell=1,2, \cdots, n$, the rank $n-\ell+1$ discrete valuation

$$
\bar{v}_{K, \geq \ell}: K \rightarrow \mathbb{Z}^{n-\ell+1} \cup\{\infty\}
$$

on $K$ induced from the rank $n$ valuation $\bar{v}_{K}$ of $K$ by the rule

$$
\bar{v}_{K, \geq \ell}(a)=\operatorname{Pr}_{\geq \ell}\left(\bar{v}_{K}(a)\right)
$$

for each $a \in K$, where

$$
\operatorname{Pr}_{\geq \ell}: \mathbb{Z}^{n} \rightarrow \mathbb{Z}^{n-\ell+1}
$$

is the projection map defined by

$$
\operatorname{Pr}_{\geq \ell}:\left(m_{1}, \cdots, m_{n}\right) \mapsto\left(m_{\ell}, \cdots, m_{n}\right)
$$

for every $\left(m_{1}, \cdots, m_{n}\right) \in \mathbb{Z}^{n}$. In particular, the rank 1 valuation $\bar{v}_{K, \geq n}$ on $K$ is nothing but the first valuation $\nu_{K_{n}}$ of $K_{n}$. Now, define a family of ideals of $K$, for each $\ell=1,2, \cdots, n$, by

$$
P_{\bar{v}_{K}}^{\left(i_{\ell}, \cdots, i_{n}\right)}:=\left\{a \in K \mid \bar{v}_{K, \geq \ell}(a) \succeq\left(i_{\ell}, \cdots, i_{n}\right)\right\}
$$

for every $\left(i_{\ell}, \cdots, i_{n}\right) \in \mathbb{Z}^{n-\ell+1}$. Observe that

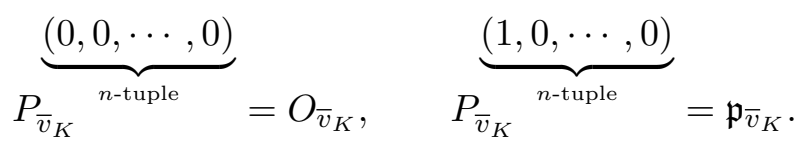

Note that, the collection of all non-zero ideals of $O_{\bar{v}_{K}}$ consists of all ideals $P_{\bar{v}_{K}}^{\left(i_{\ell}, \cdots, i_{n}\right)}$ satisfying $\left(i_{\ell}, \cdots, i_{n}\right) \succeq \overbrace{(0, \cdots, 0)}^{(n-\ell+1) \text {-tuple }}$, for each $1 \leq \ell \leq n$. Thus, we see that $O_{\bar{v}_{K}}$ is not a Noetherian ring for $n>1$.

Now, the unit group $U_{\bar{v}_{K}}$ and the group of principal units $V_{\bar{v}_{K}}$ of $K$ relative to the rank $n$ discrete valuation $\bar{v}_{K}$ are defined by

$$
U_{\bar{v}_{K}}=O_{\bar{v}_{K}}^{\times}, \quad V_{\bar{v}_{K}}=1+\mathfrak{p}_{\bar{v}_{K}} .
$$

It is also possible to define the higher-unit groups $U_{\bar{v}_{K}}^{\left(i_{\ell}, \cdots, i_{n}\right)}$ of $K$ relative to the rank $n$ discrete valuation $\bar{v}_{K}$ by

$$
U_{\bar{v}_{K}}^{\left(i_{\ell}, \cdots, i_{n}\right)}=1+P_{\bar{v}_{K}}^{\left(i_{\ell}, \cdots, i_{n}\right)}=\left\{a \in K \mid \bar{v}_{K, \geq \ell}(a-1) \succeq\left(i_{\ell}, \cdots, i_{n}\right)\right\},
$$

where $\left(i_{\ell}, \cdots, i_{n}\right) \in \mathbb{Z}^{n-\ell+1}$ satisfying $\left(i_{\ell}, \cdots, i_{n}\right) \succeq \overbrace{(0, \cdots, 0)}^{(n-\ell+1) \text {-tuple }}$, for each $\ell$ satisfying $1 \leq \ell \leq n$.

In particular, in case $\ell=n$, as already mentioned the rank 1 discrete valuation $\bar{v}_{K, \geq n}$ : $K \rightarrow \mathbb{Z} \cup\{\infty\}$ is $\nu_{K_{n}}: K_{n} \rightarrow \mathbb{Z} \cup\{\infty\}$, and in this setting:

$$
U_{\bar{v}_{K}}^{\left(i_{n}\right)}=\left\{a \in K \mid \bar{v}_{K, \geq n}(a-1) \succeq i_{n}\right\}=\left\{a \in K \mid \nu_{K_{n}}(a-1) \geq i_{n}\right\},
$$

where $i_{n} \in \mathbb{Z}$ satisfies $i_{n} \geq 0$. Thus, we shall use the standard notation for $U_{\bar{v}_{K}}^{\left(i_{n}\right)}$, and set

$$
U_{\bar{v}_{K}}^{\left(i_{n}\right)}=U_{K_{n}}^{i_{n}},
$$

for each $i_{n} \in \mathbb{Z}$ such that $i_{n} \geq 0$. Moreover, in the specific case $i_{n}=1$, we further denote the group of principal units $U_{\bar{v}_{K}}^{\left(i_{n}=1\right)}$ of $K=K_{n}$ relative to the rank 1 discrete valuation $\bar{v}_{K, \geq n}=\nu_{K_{n}}$ of $K=K_{n}$ by

$$
U_{\bar{v}_{K}}^{\left(i_{n}=1\right)}=V_{K_{n}} .
$$

Remark 2.6. The objects $O_{\bar{v}_{K}}, \mathfrak{p}_{\bar{v}_{K}}, U_{\bar{v}_{K}}, V_{\bar{v}_{K}}$, and $P_{\bar{v}_{K}}^{\left(i_{\ell}, \cdots, i_{n}\right)}, U_{\bar{v}_{K}}^{\left(i_{\ell}, \cdots, i_{n}\right)}$ introduced so far do not depend on the choice of a system of local parameters $\Pi_{K}$ of the $n$-dimensional local field $K$. 
If $\Pi_{K}=\left(t_{1, K}, \cdots, t_{n, K}\right)$ is a system of local parameters of $K$, then as in the classical 1-dimensional case, we can describe the multiplicative group $K^{\times}$of the $n$-dimensional local field $K$ by

$$
K^{\times} \simeq \mathbb{Z} t_{n, K} \oplus \mathbb{Z} t_{n-1, K} \oplus \cdots \oplus \mathbb{Z} t_{1, K} \oplus U_{\bar{v}_{K}}
$$

and

$$
U_{\bar{v}_{K}} \simeq R_{\bar{v}_{K}} \oplus V_{\bar{v}_{K}}
$$

where $R_{\bar{v}_{K}}$ is the subgroup in $K^{\times}$consisting of Teichmüller representatives of all non-zero elements of the last-residue field $K_{0}=\mathbb{F}_{q}$ of $K$. Moreover, any $a \in K$ has a unique expression as a formal power series

$$
a=\sum_{\boldsymbol{b}=\left(b_{1}, \cdots, b_{n}\right)}\left[\theta_{\boldsymbol{b}}\right] t_{1, K}^{b_{1}} \cdots t_{n, K}^{b_{n}},
$$

where all coefficients $\left[\theta_{\boldsymbol{b}}\right]$ are from the Teichmüller representatives of all non-zero elements of the last residue field $K_{0}=\mathbb{F}_{q}$ of $K$ and the summation over $\boldsymbol{b}$ runs over the admissible set $\left\{\boldsymbol{b} \in \mathbb{Z}^{n} \mid \theta_{\boldsymbol{b}} \neq 0\right\}$, which is well-ordered in $\mathbb{Z}^{n}$.

For any algebraic extension $L$ of $K$, there exists a unique extension $\bar{w}_{L}$ of the rank $n$ discrete valuation $\bar{v}_{K}$ of $K$ to $L$. Now, let in particular, $L / K$ be a finite extension. Then, $L$ has an $n$-dimensional local field structure with the corresponding Parshin chain

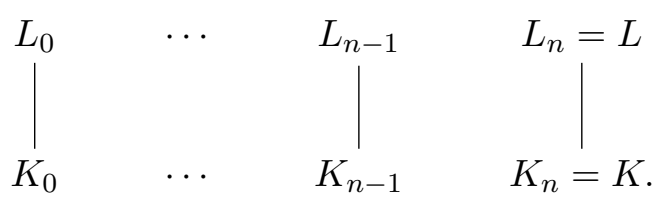

Let $\Pi_{K}=\left(t_{1, K}, \cdots, t_{n, K}\right) \in K^{n}$ and $\Pi_{L}=\left(t_{1, L}, \cdots, t_{n, L}\right) \in L^{n}$ be systems of local parameters of $K$ and of $L$ respectively. As usual, let $\bar{v}_{K}$ and $\bar{v}_{L}$ be the corresponding rank $n$ discrete valuations on $K$ and on $L$ respectively. Then, for every $a \in K \subseteq L$, the $n$-tuples

$$
\bar{v}_{K}(a):=\left(\nu_{K_{1}}, \cdots, \nu_{K_{n}}\right) \circ \rho_{\Pi_{K}}(a)
$$

and

$$
\bar{v}_{L}(a):=\left(\nu_{L_{1}}, \cdots, \nu_{L_{n}}\right) \circ \rho_{\Pi_{L}}(a)
$$

are both in $\mathbb{Z}^{n}$, and they are related by

$$
\bar{v}_{L}(a)=\bar{v}_{K}(a) E\left(L / K ; \Pi_{K}, \Pi_{L}\right),
$$

where $E\left(L / K ; \Pi_{K}, \Pi_{L}\right) \in \mathrm{M}(n, \mathbb{Z})$ is the lower-triangular integral matrix given by

$$
E\left(L / K ; \Pi_{K}, \Pi_{L}\right)=\left(v_{L j}\left(t_{i, K}\right)\right)_{i, j} .
$$

The diagonal entries of $E\left(L / K ; \Pi_{K}, \Pi_{L}\right)$ do not depend on the choice of the systems of local parameters $\Pi_{K}$ and $\Pi_{L}$. Therefore, the diagonal elements of $E\left(L / K ; \Pi_{K}, \Pi_{L}\right)$ will be denoted simply by $e_{1}(L / K), \cdots, e_{n}(L / K)$. As a notation, let $\left[L_{0}: K_{0}\right]=f(L / K)$. It is then easy to prove that

$$
\left[L_{i}: K_{i}\right]=f(L / K) e_{1}(L / K) \cdots e_{i}(L / K),
$$

for $i=1 \cdots, n$, where

$$
e_{\ell}(L / K)=e\left(L_{\ell} / K_{\ell}\right)
$$

for $\ell=1, \cdots, i \leq n$. Moreover, the finite extension $L / K$ is called:

- totally ramified, if $f(L / K)=1$ (or equivalently $L_{0}=K_{0}$ );

- semi ramified if $e_{n}(L / K)=1$ and $L_{n-1} / K_{n-1}$ is separable;

- purely unramified, if the equality $[L: K]=f(L / K)$ (or equivalently $\prod_{i=1}^{n} e_{i}(L / K)=$ 1) holds. 
If $K$ satisfies $\operatorname{char}\left(K_{n-1}\right)=p>0$, then $\left[L_{n-1}: K_{n-1}\right]$ has an expression of the form

$$
\left[L_{n-1}: K_{n-1}\right]=f_{0} \cdot p^{s},
$$

where $f_{0}$ is the separable degree of $L_{n-1} / K_{n-1}$ denoted by $f_{0}(L / K)$, and $p^{s}$ is the inseparable degree of $L_{n-1} / K_{n-1}$ denoted by $s(L / K)$.

Now, assume that $L / K$ is an infinite algebraic extension in a fixed algebraic closure $\bar{K}$. The infinite algebraic extension $L / K$ is called:

- totally ramified, if every finite subextension $F / K$ of $L / K$ inside $\bar{K}$ is totally ramified. Thus, if $M / K$ is any subextension of a totally ramified extension $L / K$, then $M / K$ is totally ramified as well. Moreover, $L / K$ is called maximal totally ramified, if there is no totally ramified extension $E / K$ satisfying $L \subsetneq E \subset \bar{K}$. A maximal totally ramified extension of $K$ in $\bar{K}$ exists but it is not unique. Note that, the compositum of a collection of totallly ramified extensions of $K$ inside $\bar{K}$ is not necessarily totally ramified over $K$.

- purely unramified, if every finite subextension $F / K$ of $L / K$ inside $\bar{K}$ is purely unramified. Thus, if $M / K$ is any subextension of a purely unramified extension $L / K$, then $M / K$ is purely unramified as well. The compositum of a collection of purely unramified extensions of $K$ in $\bar{K}$ is again purely unramified over $K$. Therefore, the compositum $K^{\text {pur }}$ of all purely unramified extensions of $K$ in $\bar{K}$ is the maximal purely unramified extension of $K$ in $\bar{K}$. Moreover,

$$
K^{\text {pur }}=\bigcup_{(m, p)=1} K\left(\zeta_{m}\right),
$$

where $\zeta_{m}$ is a primitive $m^{\text {th }}$ root of unity with $m$ relatively prime to $p$. Thus, it follows that $K^{\text {pur }}$ is Galois over $K$. A topological generator $\varphi_{K}$ of $\operatorname{Gal}\left(K^{\text {pur }} / K\right)$ which is mapped on the topological generator $\operatorname{Frob}_{q}$ of $\operatorname{Gal}\left(\mathbb{F}_{q}^{\text {sep }} / \mathbb{F}_{q}\right)$ is called the Frobenius automorphism of $K$. So, for each $0<d \in \mathbb{Z}$, there exists a unique purely unramified extension $K^{\text {pur,d }}$ of degree $d$ over $K$, which is the splitting field of the polynomial $X^{p^{d}}-X \in K[X]$ over $K$. Moreover, note that, if $L / K$ is purely unramified, and $\Pi_{K} \in K^{n}$ is a system of local parameters of $K$, then $\Pi_{K} \in L^{n}$ remains a system of local parameters of $L$ as well.

The proof of the following proposition is clear.

Proposition 2.7. If $L / K$ is any algebraic extension, then its unique maximal purely unramified subextension $L_{o} / K$ is nothing but $L_{o}=L \cap K^{\text {pur }}$.

Moreover,

Proposition 2.8. Let $L / K$ be a finite extension. Then the unique maximal purely unramified subextension $L_{o} / K$ of $L / K$ is the splitting field of the polynomial $X^{p^{f(L / K)}}-X \in K[X]$ over $K$. Moreover, $L / L_{o}$ is a totally ramified extension, and

$$
\left[L: L_{o}\right]=e_{1}(L / K) \cdots e_{n}(L / K), \quad\left[L_{o}: K\right]=f(L / K) .
$$

A special case of this proposition reads as follows: Let $L / K$ be an algebraic extension. Then,

$$
L / K \text { : totally ramified } \Leftrightarrow L_{o}=K \text {. }
$$

\section{Topologies on an $n$-dimensional local field}

There are several topologies related to the $n$-dimensional local field $K$ with the corresponding Parshin chain

$$
K_{0}, K_{1}, \cdots, K_{n-1}, K_{n}=K .
$$


- The complete discrete valuation $\nu_{K_{n}}: K_{n} \rightarrow \mathbb{Z} \cup\{\infty\}$ on $K_{n}$ defines a natural topology on $K_{n}=K$, called the discrete valuation topology on $K$, denoted by $\mathscr{V}_{K_{n}}$. With respect to $\mathscr{V}_{K_{n}}$ :

- $K$ has a natural complete and Hausdorff topological field structure;

- As a topological field, $K$ is not locally compact in case $n \geq 2$ as $\kappa_{K_{n}}=K_{n-1}$ is not a finite field;

- Moreover, again in case $n \geq 2$, the elements of $K$, which can be considered as formal series $\sum_{i} a_{i} t_{n, K}^{i}$ in the first local parameter $t_{n, K}$ of $K$ via the structure theorem of higher-dimensional local fields, do not converge, as $\left|a_{i}\right|_{\nu_{K_{n}}}=1$ whenever $a_{i} \neq 0$.

Let $\Pi_{K}=\left(t_{1, K}, \cdots, t_{n, K}\right) \in K^{n}$ be a system of local parameters of $K$ and $\bar{v}_{K}: K \rightarrow$ $\mathbb{Z}^{n} \cup\{\infty\}$ be the corresponding rank $n$ discrete valuation on $K$ introduced in Section 2 .

- There is a natural topology $\mathscr{T}_{K}$ on $K$, called the higher topology on $K$, which is defined recursively by the higher topologies on the residue fields $K_{n-1}, \cdots, K_{1}$ and $K_{0}$, where the higher topology $\mathscr{T}_{K_{1}}$ on $K_{1}$ coincides with the discrete valuation topology $\mathscr{V}_{K_{1}}$ on $K_{1}$, look at [50] for details. With respect to the topology $\mathscr{T}_{K}$ :

- $K$ does not have a topological field structure. In fact, $K$ is a complete and Hausdorff sequential ring; that is, the additive group $K^{+}$is a topological group, multiplication $K \times K \stackrel{\times}{\rightarrow} K$ on $K$ is sequentially continuous. In general, the inversion $K^{\times} \stackrel{\iota}{\rightarrow} K^{\times}$on $K^{\times}$is not sequentially continuous with respect to the induced topology of $\mathscr{T}_{K}$ on $K^{\times}$. Look at $[5,6]$ for an overview of sequential algebraic structures;

- The map $K \rightarrow K$ defined by multiplication with a fixed non-zero $a_{o} \in K$ as $a \mapsto a_{o} . a$ for every $a \in K$ is a homeomorphism;

- The residue homomorphism $O_{\bar{v}_{K}} \rightarrow K_{n-1}$ is continuous and open, where $O_{\bar{v}_{K}}$ is equipped with the subspace topology induced from the higher topology $\mathscr{T}_{K}$ of $K$ and $K_{n-1}$ is endowed with its higher topology $\mathscr{T}_{K_{n-1}}$;

- The unique formal power series expression of $a \in K$ given by

$$
a=\sum_{\boldsymbol{b}=\left(b_{1}, \cdots, b_{n}\right)}\left[\theta_{\boldsymbol{b}}\right] t_{1, K}^{b_{1}} \cdots t_{n, K}^{b_{n}},
$$

where all coefficients $\left[\theta_{\boldsymbol{b}}\right]$ are from the Teichmüller representatives of all nonzero elements of the last residue field $K_{0}=\mathbb{F}_{q}$ of $K$ and the summation is over the admissible well-ordered set $\left\{\boldsymbol{b} \in \mathbb{Z}^{n} \mid \theta_{\boldsymbol{b}} \neq 0\right\}$, is absolutely convergent.

- There is also a natural topology $\mathscr{T}_{K \times}$ on the multiplicative group $K^{\times}$, called the higher topology on $K^{\times}$, which is defined as the initial (that is, weakest) topology on $K^{\times}$that makes the map

$$
K^{\times} \rightarrow K \times K
$$

given by

$$
a \mapsto\left(a, a^{-1}\right),
$$

for every $a \in K^{\times}$sequentially continuous. Equivalently, the topology $\mathscr{T}_{K^{\times}}$on $K^{\times}$ is defined as follows:

If $\operatorname{char}\left(K_{n-1}\right)=p>0$, then the topology $\mathscr{T}_{K^{\times}}$on $K^{\times}$is defined to be the unique topology on $K^{\times}$that turns the isomorphism

$$
K^{\times} \stackrel{\sim}{\rightarrow}\left\langle t_{n, K}\right\rangle \times \cdots \times\left\langle t_{1, K}\right\rangle \times R_{\bar{v}_{K}} \times V_{\bar{v}_{K}}
$$

into a topological group isomorphism. Here, as introduced in the previous section, $R_{\bar{v}_{K}}$ is the subgroup of $K^{\times}$consisting of Teichmüller representatives of all non-zero elements of the last-residue field $K_{0}=\mathbb{F}_{q}$ of $K, V_{\bar{v}_{K}}$ is the group of principal units of $K$ relative to $\bar{v}_{K}$, and the topology on $\left\langle t_{n, K}\right\rangle \times \cdots \times\left\langle t_{1, K}\right\rangle \times R_{\bar{v}_{K}} \times V_{\bar{v}_{K}}$ is the 
product topology defined by the discrete topology on $\left\langle t_{n, K}\right\rangle \times \cdots \times\left\langle t_{1, K}\right\rangle \times R_{\bar{v}_{K}}$ and the topology on $V_{\bar{v}_{K}}$ induced from the topology $\mathscr{T}_{K}$ on $K$.

If $\operatorname{char}(K)=\cdots=\operatorname{char}\left(K_{m+1}\right)=0, \operatorname{char}\left(K_{m}\right)=p>0$ for some $m \leq n-2$, then the natural topology $\mathscr{T}_{K} \times$ on $K^{\times}$is defined to be the unique topology on $K^{\times}$ that turns the isomorphism

$$
K^{\times} \stackrel{\sim}{\rightarrow}\left\langle t_{n, K}\right\rangle \times \cdots \times\left\langle t_{1, K}\right\rangle \times R_{\bar{v}_{K}} \times V_{\bar{v}_{K}}
$$

into a topological group isomorphism, where the topology on $\left\langle t_{n, K}\right\rangle \times \cdots \times\left\langle t_{1, K}\right\rangle \times$ $R_{\bar{v}_{K}} \times V_{\bar{v}_{K}}$ is the product topology defined by the discrete topology on $\left\langle t_{n, K}\right\rangle \times \cdots \times$ $\left\langle t_{1, K}\right\rangle$ and the topology on $U_{\bar{v}_{K}}=R_{\bar{v}_{K}} \times V_{\bar{v}_{K}}$ induced from the natural subspace topology on $U_{\bar{v}_{K_{m+1}}}$ given by $\mathscr{T}_{K_{m+1}^{\times}}$via the canonical short exact sequence

$$
1 \rightarrow 1+P_{\bar{v}_{K}}^{(1,0, \cdots, 0)} \rightarrow U_{\bar{v}_{K}} \rightarrow U_{\bar{v}_{K_{m+1}}} \rightarrow 1 .
$$

The basic properties of the topology $\mathscr{T}_{K \times}$ on $K^{\times}$are the following :

- Every Cauchy sequence in $K^{\times}$with respect to the topology $\mathscr{T}_{K^{\times}}$converges in $K^{\times}$;

- Multiplication $K^{\times} \times K^{\times} \stackrel{\times}{\rightarrow} K^{\times}$on $K^{\times}$is sequentially continuous and the inversion $K^{\times} \stackrel{\iota}{\rightarrow} K^{\times}$on $K^{\times}$is sequentially continuous. That is, $K^{\times}$becomes a sequential group;

- If $n \leq 2$, then the multiplicative group $K^{\times}$is furthermore a topological group with respect to $\mathscr{T}_{K \times}$ with a countable base of open subgroups. If $n \geq 3$, then the multiplicative group $K^{\times}$is not a topological group with respect to $\mathscr{T}_{K^{\times}}$;

- The unique formal product expression of $a \in K^{\times}$given by

$$
a=t_{1, K}^{r_{1}} \cdots t_{n, K}^{r_{n}} \theta \prod_{\boldsymbol{b}=\left(b_{1}, \cdots, b_{n}\right)}\left(1+\left[\theta_{\boldsymbol{b}}\right] t_{1, K}^{b_{1}} \cdots t_{n, K}^{b_{n}}\right),
$$

where $r_{1}, \cdots, r_{n} \in \mathbb{Z}$, all coefficients $\left[\theta_{\boldsymbol{b}}\right]$ and $\theta$ are from the Teichmüller representatives of all non-zero elements of the last residue field $K_{0}=\mathbb{F}_{q}$ of $K$ and the product is over the admissible well-ordered set $\left\{\boldsymbol{b} \in \mathbb{Z}^{n} \mid \theta_{\boldsymbol{b}} \neq 0\right\}$, is absolutely convergent.

For details about the topology $\mathscr{T}_{K^{\times}}$, look at [50].

As Fesenko points out, the higher topology $\mathscr{T}_{K}$ on $K$ and the higher topology $\mathscr{T}_{K \times}$ on $K^{\times}$ are indeed "the appropriate topologies" for class field theoretic investigations for $K$. It is also quite possible, as suggested by Braunling, that a totally new theory, like "condensed mathematics" of Clausen and Scholze [46] or "pyknotic mathematics" of Barwick and Haine [3], is needed to settle the topological problems of $K$.

\section{Milnor $K$-theory}

Let $F$ be any field. For any integer $m>0$, the $m^{\text {th }}$ Milnor $K$-group $\mathrm{K}_{m}^{\text {Milnor }}(F)$ of $F$ is defined by the quotient

$$
\mathrm{K}_{m}^{\mathrm{Milnor}}(F):=F^{\times \otimes m} / J_{m}(F),
$$

where $F^{\times \otimes m}=\overbrace{F^{\times} \otimes \cdots \otimes F^{\times}}^{m \text {-copies }}$ is the $m$-fold tensor product of $F^{\times}$and $J_{m}(F)$ is the subgroup of $F^{\times \otimes m}$ defined by

$$
\left\langle x_{1} \otimes \cdots \otimes x_{m} \mid x_{i}+x_{j}=1,{ }^{\exists} i, j, 1 \leq i \neq j \leq m\right\rangle .
$$

For $x_{1}, \cdots, x_{m} \in F^{\times}$, the element $x_{1} \otimes \cdots \otimes x_{m}\left(\bmod J_{m}(F)\right)$ in $\mathrm{K}_{m}^{\mathrm{Milnor}}(F)$ is simply denoted by $\left\{x_{1}, \cdots, x_{m}\right\}$ and called the generalized Steinberg symbol of $x_{1}, \cdots, x_{m}$. In case $m=0$, we set $\mathrm{K}_{m=0}^{\text {Milnor }}(F)=\mathbb{Z}$. 
Milnor $K$-theory $\mathrm{K}_{m}^{\text {Milnor }}$ defines a functor from the category of fields to the category of abelian groups. Let $L / F$ be any extension. Then the natural embedding $j_{L / F}: F \hookrightarrow L$ induces a group homomorphism

$$
\mathrm{K}_{m}^{\text {Milnor }}\left(j_{L / F}\right)=j_{L / F}^{\text {Milnor }}: \mathrm{K}_{m}^{\text {Milnor }}(F) \rightarrow \mathrm{K}_{m}^{\text {Milnor }}(L) .
$$

In case $m=0$, the homomorphism $j_{L / F}^{\text {Milnor }}$ is the identity arrow $\operatorname{id}_{\mathbb{Z}}: \mathbb{Z} \rightarrow \mathbb{Z}$.

By a theorem of Bass, Tate and Kato, there exists, for each finite extension $L / F$, a group homomorphism

$$
\mathrm{N}_{L / F}^{\mathrm{Milnor}}: \mathrm{K}_{m}^{\mathrm{Milnor}}(L) \rightarrow \mathrm{K}_{m}^{\mathrm{Milnor}}(F)
$$

called the (K-theoretic) norm map from $L$ to $F$. The basic properties of this arrow are the following:

- The norm map $\mathrm{N}_{L / F}^{\text {Milnor }}: \mathrm{K}_{m}^{\mathrm{Milnor}}(L) \rightarrow \mathrm{K}_{m}^{\mathrm{Milnor}}(F)$ from $L$ to $F$ is transitive in the sense that, for every chain $F \subset M \subset L$ of extensions of $F$, the equality

$$
\mathrm{N}_{L / F}^{\mathrm{Milnor}}=\mathrm{N}_{M / F}^{\mathrm{Milnor}} \circ \mathrm{N}_{L / M}^{\mathrm{Milnor}}
$$

holds;

- In the low-dimensional cases, the homomorphism

$$
\mathrm{N}_{L / F}^{\text {Milnor }}: \mathrm{K}_{m}^{\text {Milnor }}(L) \rightarrow \mathrm{K}_{m}^{\text {Milnor }}(F)
$$

reduces to the multiplication by $[L: F]$ mapping if $m=0$, and to the usual norm map of fields $\mathrm{N}_{L / F}: L^{\times} \rightarrow F^{\times}$if $m=1$;

- The composition

$$
\mathrm{K}_{m}^{\text {Milnor }}(F) \stackrel{j_{L / F}^{\text {Milnor }}}{\longrightarrow} \mathrm{K}_{m}^{\text {Milnor }}(L) \stackrel{\mathrm{N}_{L / F}^{\text {Milnor }}}{\longrightarrow} \mathrm{K}_{m}^{\text {Milnor }}(F)
$$

is the mapping defined as the multiplication by $[L: F]$;

- If $\sigma \in \operatorname{Aut}_{F}(L)$, then

$$
\mathrm{N}_{L / F}^{\text {Milnor }} \circ \mathrm{K}_{m}^{\text {Milnor }}(\sigma)=\mathrm{N}_{L / F}^{\text {Milnor }},
$$

where $\mathrm{K}_{m}^{\text {Milnor }}(\sigma): \mathrm{K}_{m}^{\text {Milnor }}(L) \rightarrow \mathrm{K}_{m}^{\text {Milnor }}(L)$ is the homomorphism induced by the $F$-automorphism $\sigma: L \rightarrow L$.

For details about Milnor $K$-theory, look at Chapter IX of [15].

In case, $K$ is the $n$-dimensional local field with the corresponding Parshin chain

$$
\mathbb{F}_{q}=K_{0}, K_{1}, \cdots, K_{n-1}, K_{n}=K,
$$

there exists a surjective homomorphism called the (K-theoretic) valuation map

$$
\nu_{\mathrm{K}_{n}^{\mathrm{Milnor}}(K)}: \mathrm{K}_{n}^{\mathrm{Milnor}}(K) \rightarrow \mathbb{Z}
$$

on $\mathrm{K}_{n}^{\mathrm{Milnor}}(K)$ defined by the composition

$$
\nu_{\mathrm{K}_{n}^{\mathrm{Milnor}}(K)}: \mathrm{K}_{n}^{\mathrm{Milnor}}\left(K_{n}\right) \stackrel{\partial_{n-1}^{n}}{\longrightarrow} \mathrm{K}_{n-1}^{\mathrm{Milnor}}\left(K_{n-1}\right) \stackrel{\partial_{n-2}^{n-1}}{\longrightarrow} \cdots \stackrel{\partial_{0}^{1}}{\longrightarrow} \mathrm{K}_{0}^{\mathrm{Milnor}}\left(K_{0}\right)=\mathbb{Z},
$$

where the arrows

$$
\partial_{i-1}^{i}: \mathrm{K}_{i}^{\mathrm{Milnor}}\left(K_{i}\right) \rightarrow \mathrm{K}_{i-1}^{\mathrm{Milnor}}\left(K_{i-1}\right)
$$

for $i=1,2,3, \cdots, n$, are the boundary homomorphisms in Milnor K-theory defined by

$$
\partial_{i-1}^{i}\left(\left\{u_{1}, \cdots, u_{i-1}, x\right\}\right)=\nu_{K_{i}}(x)\left\{\bar{u}_{1}, \cdots, \bar{u}_{i-1}\right\}
$$

for each $u_{1}, \cdots, u_{i-1} \in O_{K_{i}}^{\times}=U_{K_{i}}$ and $x \in K_{i}^{\times}$, where $\bar{u}_{1}, \cdots, \bar{u}_{i-1} \in K_{i-1}$ are defined by reduction modulo $\mathfrak{p}_{K_{i}}$ of the elements $u_{1}, \cdots, u_{i-1}$ in $K_{i}$. Let $L$ be a finite extension of $K$. Then the $K$-theoretic valuation map

$$
\nu_{\mathrm{K}_{n}^{\mathrm{Milnor}}(L)}: \mathrm{K}_{n}^{\mathrm{Milnor}}(L) \rightarrow \mathbb{Z}
$$


on $\mathrm{K}_{n}^{\text {Milnor }}(L)$ satisfies

$$
\nu_{\mathrm{K}_{n}^{\text {Milnor }}(L)}=\frac{1}{f(L / K)} \nu_{\mathrm{K}_{n}^{\text {Milnor }}(K)} \circ \mathrm{N}_{L / K}^{\text {Milnor }},
$$

where $f(L / K)=\left[L_{0}: K_{0}\right]$, because the diagram

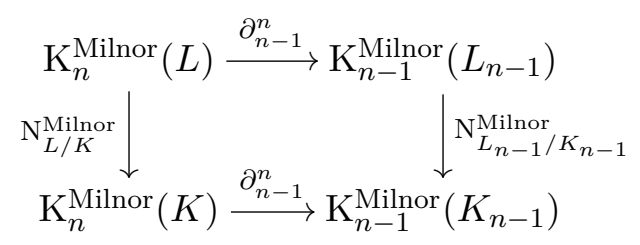

is commutative. An element $\Pi_{K_{n}^{\text {Milnor }}(K)}$ of $\mathrm{K}_{n}^{\text {Milnor }}(K)$ is called a prime element of $\mathrm{K}_{n}^{\mathrm{Milnor}}(K)$ if

$$
\nu_{\mathrm{K}_{n}^{\mathrm{Milnor}}(K)}\left(\Pi_{\mathrm{K}_{n}^{\mathrm{Milnor}}(K)}\right)=1 .
$$

Note that, a prime element $\Pi_{\mathrm{K}_{n}^{\mathrm{Milnor}}(K)}$ of $\mathrm{K}_{n}^{\mathrm{Milnor}}(K)$ can be expressed as

$$
\Pi_{\mathrm{K}_{n}^{\mathrm{Milnor}}(K)}=\left\{t_{1, K}, \cdots, t_{n, K}\right\}+\varepsilon,
$$

where $\Pi_{K}=\left(t_{1, K}, \cdots, t_{n, K}\right) \in K^{n}$ is a system of local parameters of the $n$-dimensional local field $K$ and the element $\varepsilon$ lies in $\operatorname{Ker}\left(\nu_{\mathrm{K}_{n}^{\mathrm{Mi}} \text { inor }(K)}\right)$.

Continue to assume that $K$ is an $n$-dimensional local field. Then, choosing a system of local parameters $\Pi_{K}=\left(t_{1, K}, \cdots, t_{n, K}\right) \in K^{n}$ of $K, \Pi_{K}$ determines a rank $n$ discrete valuation

$\bar{v}_{K}: K \rightarrow \mathbb{Z}^{n} \cup\{\infty\}$
of $K$, which determines a collection $\left\{U_{\bar{v}_{K}}^{\left(i_{\ell}, \cdots, i_{n}\right)} \mathrm{K}_{m}^{\mathrm{Milnor}}(K)\right\}_{\left(i_{\ell}, \cdots, i_{n}\right)}$ consisting of subgroups $U_{\bar{v}_{K}}^{\left(i_{\ell}, \cdots, i_{n}\right)} \mathrm{K}_{m}^{\mathrm{Milnor}}(K)$ of $\mathrm{K}_{m}^{\mathrm{Milnor}}(K)$ given by

$$
U_{\bar{v}_{K}}^{\left(i_{\ell}, \cdots, i_{n}\right)} \mathrm{K}_{m}^{\mathrm{Milnor}}(K)=\left\langle\left\{x_{1}, \cdots, x_{m}\right\} \in \mathrm{K}_{m}^{\mathrm{Milnor}}(K) \mid x_{1} \in U_{\bar{v}_{K}}^{\left(i_{\ell}, \cdots, i_{n}\right)}\right\rangle,
$$

where $\left(i_{\ell}, \cdots, i_{n}\right) \in \mathbb{Z}^{n-\ell+1}$ satisfies $\left(i_{\ell}, \cdots, i_{n}\right) \succeq \overbrace{(0, \cdots, 0)}^{(n-\ell+1) \text {-tuple }}$, for each $\ell$ satisfying $1 \leq \ell \leq n$.

In particular, in case $\ell=n$, the group $U_{\bar{v}_{K}}^{\left(i_{n}\right)} \mathrm{K}_{m}^{\mathrm{Milnor}}(K)$ is denoted by $U_{K_{n}}^{i_{n}} \mathrm{~K}_{m}^{\mathrm{Milnor}}(K)$ for each $i_{n} \in \mathbb{Z}$ satisfying $i_{n} \geq 0$. Moreover,

- if $i_{n}=0$, the group $U_{\bar{v}_{K}}^{\left(i_{n}=0\right)} \mathrm{K}_{m}^{\mathrm{Milnor}}(K)$ is denoted by $U_{K_{n}} \mathrm{~K}_{m}^{\mathrm{Milnor}}(K)$;

- if $i_{n}=1$, the group $U_{\bar{v}_{K}}^{\left(i_{n}=1\right)} \mathrm{K}_{m}^{\text {Milnor }}(K)$ is denoted by $V_{K_{n}} \mathrm{~K}_{m}^{\text {Milnor }}(K)$.

In case $L$ is an algebraic extension of $K$ and $\bar{w}_{L}$ is the unique extension of the rank $n$ discrete valuation $\bar{v}_{K}$ of $K$ to $L$, the subgroup

$$
U_{\bar{w}_{L}}^{\left(i_{\ell}, \cdots, i_{n}\right)} \mathrm{K}_{m}^{\mathrm{Milnor}}(L)=\left\langle\left\{x_{1}, \cdots, x_{m}\right\} \in \mathrm{K}_{m}^{\mathrm{Milnor}}(L) \mid x_{1} \in U_{\bar{w}_{L}}^{\left(i_{\ell}, \cdots, i_{n}\right)}\right\rangle,
$$

of $\mathrm{K}_{m}^{\mathrm{Milnor}}(L)$ is denoted by $U_{\bar{v}_{K}}^{\left(i_{\ell}, \cdots, i_{n}\right)} \mathrm{K}_{m}^{\mathrm{Milnor}}(L)$, where $\left(i_{\ell}, \cdots, i_{n}\right) \in \mathbb{Z}^{n-\ell+1}$ satisfies $(n-\ell+1)$-tuple

$\left(i_{\ell}, \cdots, i_{n}\right) \succeq \overbrace{(0, \cdots, 0)}$, for each $\ell$ satisfying $1 \leq \ell \leq n$.

In particular, in case $\ell=n$, the group $U_{\bar{v}_{K}}^{\left(i_{n}\right)} \mathrm{K}_{m}^{\text {Milnor }}(L)$ is denoted by $U_{K_{n}}^{i_{n}} \mathrm{~K}_{m}^{\text {Milnor }}(L)$ for each $i_{n} \in \mathbb{Z}$ satisfying $i_{n} \geq 0$. Moreover,

- if $i_{n}=0$, the group $U_{\bar{v}_{K}}^{\left(i_{n}=0\right)} \mathrm{K}_{m}^{\mathrm{Milnor}}(L)$ is denoted by $U_{K_{n}} \mathrm{~K}_{m}^{\text {Milnor }}(L)$;

- if $i_{n}=1$, the group $U_{\bar{v}_{K}}^{\left(i_{n}=1\right)} \mathrm{K}_{m}^{\text {Milnor }}(L)$ is denoted by $V_{K_{n}} \mathrm{~K}_{m}^{\text {Milnor }}(L)$. 


\section{5. $\mathrm{K}_{*}^{\text {top }}$-groups}

Let $F$ be a field such that $F^{\times}$is endowed with a topology $\mathscr{T}$. The topology $\mathscr{T}$ on $F^{\times}$introduces a natural topology $\mathscr{T}_{\mathrm{K}_{m}^{\mathrm{Milnor}}(F)}$ on $\mathrm{K}_{m}^{\mathrm{Milnor}}(F)$. The sequential saturation" $\left(\mathscr{T}_{\mathrm{K}_{m}^{\mathrm{Milnor}}(F)}\right)_{\mathrm{seq}}$ of $\mathscr{T}_{\mathrm{K}_{m}^{\mathrm{Milnor}}(F)}$ is the strongest topology on $\mathrm{K}_{m}^{\mathrm{Milnor}}(F)$ that makes the mappings

and

$$
(\alpha, \beta) \mapsto \alpha-\beta,{ }^{\forall} \alpha, \beta \in \mathrm{K}_{m}^{\mathrm{Milnor}}(F)
$$

$$
\left(a_{1}, \cdots, a_{m}\right) \mapsto\left\{a_{1}, \cdots, a_{m}\right\},{ }^{\forall} a_{1}, \cdots, a_{m} \in F^{\times}
$$

continuous. Look at Remark 1 in [14]. With respect to the topology $\left(\mathscr{T}_{\mathrm{K}_{m}^{\mathrm{Milnor}}(F)}\right)_{\mathrm{seq}}$ defined on $\mathrm{K}_{m}^{\mathrm{Milnor}}(F)$, Parshin introduced

$$
\Lambda_{\mathrm{K}_{m}^{\mathrm{Milnor}}(F)}:=\bigcap_{\mathscr{O}} \mathscr{O},
$$

where $\mathscr{O}$ runs over all open neighbourhoods of the identity element $0_{K_{m}^{\mathrm{Milnor}}(F)}$ of $K_{m}^{\mathrm{Milnor}}(F)$, which is a closed subgroup of $\mathrm{K}_{m}^{\mathrm{Milnor}}(F)$. The quotient group

$$
\mathrm{K}_{m}^{\mathrm{top}}(F):=\mathrm{K}_{m}^{\mathrm{Milnor}}(F) / \Lambda_{\mathrm{K}_{m}^{\mathrm{Milnor}}(F)}
$$

endowed with the quotient topology of $\left(\mathscr{T}_{\mathrm{K}_{m}^{\mathrm{Milnor}}(F)}\right)_{\text {seq }}$; that is, the maximal Hausdorff quotient of $\mathrm{K}_{m}^{\text {Milnor }}(F)$ with respect to $\left(\mathscr{T}_{\mathrm{K}_{m}^{\mathrm{Milnor}}(F)}\right)_{\text {seq }}$, is called the $m^{\text {th }}$ Parshin topological $K$-group of the field $F$. For $x_{1}, \cdots, x_{m} \in F^{\times}$, the element $\left\{x_{1}, \cdots, x_{m}\right\}\left(\bmod \Lambda_{K_{m}^{\text {Milnor }}(F)}\right)$ is denoted by $\left\{x_{1}, \cdots, x_{m}\right\}^{\text {top }}$ and called the topological Steinberg symbol of $x_{1}, \cdots, x_{m}$.

Let $L$ be any "compatible" extension of $F$ in the sense that:

$-L^{\times}$is endowed with a topology $\mathscr{T}^{\prime}$;

- The topology $\mathscr{T}$ on $F^{\times}$is induced from $\mathscr{T}^{\prime}$.

Then, the inclusion $j_{L / F}^{\text {Milnor }}\left(\Lambda_{\mathrm{K}_{m}^{\text {Milnor }}(F)}\right) \subseteq \Lambda_{\mathrm{K}_{m}^{\text {Milnor }}(L)}$ clearly follows, and the group homomorphism $j_{L / F}^{\text {Milnor }}: \mathrm{K}_{m}^{\text {Milnor }}(F) \rightarrow \mathrm{K}_{m}^{\text {Milnor }}(L)$ extends uniquely to a continuous homomorphism

$$
j_{L / F}^{\text {top }}: \mathrm{K}_{m}^{\mathrm{top}}(F) \rightarrow \mathrm{K}_{m}^{\mathrm{top}}(L) .
$$

For the $n$-dimensional local field $K$, let $\mathscr{T}_{K \times}$ be the higher topology on $K^{\times}$introduced in Section 3. As in the preceding paragraph, the strongest topology on $\mathrm{K}_{m}^{\mathrm{Milnor}}(K)$ that makes the mappings

$$
(\alpha, \beta) \mapsto \alpha-\beta,{ }^{\forall} \alpha, \beta \in \mathrm{K}_{m}^{\mathrm{Milnor}}(K)
$$

$\|_{\text {Let }} X$ be a set endowed with a topology $\mathscr{T}$. Recall that $U \subseteq X$ is called sequentially open (with respect to $\mathscr{T})$, if for any sequence $\left(x_{n}\right)$ in $X$ converging to $u \in U$, there exists $n_{o}$ such that $x_{n} \in U$ for every $n \geq n_{o}$. The collection of sequentially open subsets of $X$ (with respect to $\mathscr{T}$ ) defines a topology $\mathscr{T}_{\text {seq }}$ on $X$ finer than $\mathscr{T}$, called the sequential saturation of $\mathscr{T}$, and the topological space $\left(X, \mathscr{T}_{\text {seq }}\right)$ the sequential saturation of the topological space $(X, \mathscr{T})$. To simplify the notation, the sequential saturation of the topological space $X$ is simply denoted by $X_{\text {seq }}$. If $X=X_{\text {seq }}$ (that is, if $\mathscr{T}=\mathscr{T}_{\text {seq }}$ ), then $X$ is called a sequentially saturated topological space. Note that, the topological space $X_{\text {seq }}$ has the following basic properties:

$-X_{\text {seq }}$ is sequentially saturated, namely $\left(X_{\text {seq }}\right)_{\text {seq }}=X_{\text {seq }}$;

- The universal mapping property satisfied by $X_{\text {seq }}$ : If $Y$ is a sequentially saturated space, then any continuous map $f: Y \rightarrow X$ factors naturally as

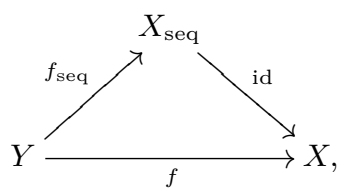

where the induced map $f_{\text {seq }}: Y \rightarrow X_{\text {seq }}$ is continuous;

- If $Y$ is a topological space and $f: Y \rightarrow X$ is any sequentially continuous map, then the induced map $f_{\text {seq }}: Y_{\text {seq }} \rightarrow X_{\text {seq }}$ is continuous. 
and

$$
\left(a_{1}, \cdots, a_{m}\right) \mapsto\left\{a_{1}, \cdots, a_{m}\right\},{ }^{\forall} a_{1}, \cdots, a_{m} \in K^{\times}
$$

continuous is the sequential saturation $\left(\mathscr{T}_{\mathrm{K}_{m}^{\mathrm{Milnor}}(K)}\right)_{\text {seq }}$ of the topology $\mathscr{T}_{\mathrm{K}}^{\mathrm{Milnor}(K)}$ on $\mathrm{K}_{m}^{\mathrm{Milnor}}(K)$, where $\mathscr{T}_{\mathrm{K}_{m}^{\mathrm{Milnor}}(K)}$ denotes the topology on $\mathrm{K}_{m}^{\mathrm{Milnor}}(K)$ induced from the higher topology $\mathscr{T}_{K^{\times}}$of $K^{\times}$.

Note that, by [14], the closed subgroup $\Lambda_{\mathrm{K}_{m}^{\mathrm{Milnor}}(K)}$ of $\mathrm{K}_{m}^{\mathrm{Milnor}}(K)$ is also equal to

$$
\Lambda_{\mathrm{K}_{m}^{\mathrm{Milnor}}(K)}=\bigcap_{\ell \neq p} \ell \mathrm{K}_{m}^{\mathrm{Milnor}}(K),
$$

where $\ell$ runs over all primes different than $p=\operatorname{char}\left(K_{0}\right)$. Therefore, the boundary homomorphism in Milnor $K$-theory

$$
\partial_{i-1}^{i}: \mathrm{K}_{i}^{\mathrm{Milnor}}\left(K_{i}\right) \rightarrow \mathrm{K}_{i-1}^{\mathrm{Milnor}}\left(K_{i-1}\right)
$$

naturally induces the following morphism

$$
\partial_{i-1}^{i}: \Lambda_{\mathrm{K}_{i}^{\mathrm{Milnor}}\left(K_{i}\right)} \rightarrow \Lambda_{\mathrm{K}_{i-1}^{\mathrm{Milnor}}\left(K_{i-1}\right)},
$$

and thereby defines the boundary homomorphism in topological Milnor K-theory

$$
\left(\partial_{i-1}^{i}\right)^{\text {top }}: \mathrm{K}_{i}^{\mathrm{top}}\left(K_{i}\right) \rightarrow \mathrm{K}_{i-1}^{\mathrm{top}}\left(K_{i-1}\right),
$$

where

$$
\begin{aligned}
\left(\partial_{i-1}^{i}\right)^{\mathrm{top}}\left(\left\{u_{1}, \cdots, u_{i-1}, x\right\}^{\mathrm{top}}\right) & =\left(\partial_{i-1}^{i}\right)^{\mathrm{top}}\left(\left\{u_{1}, \cdots, u_{i-1}, x\right\} \quad\left(\bmod \Lambda_{\mathrm{K}_{i}^{\mathrm{Milnor}}\left(K_{i}\right)}\right)\right) \\
& =\nu_{K_{i}}(x)\left\{\bar{u}_{1}, \cdots, \bar{u}_{i-1}\right\} \quad\left(\bmod \Lambda_{\mathrm{K}_{i-1}^{\mathrm{Minhor}}\left(K_{i-1}\right)}\right) \\
& =\nu_{K_{i}}(x)\left\{\bar{u}_{1}, \cdots, \bar{u}_{i-1}\right\}^{\mathrm{top}}
\end{aligned}
$$

for each $u_{1}, \cdots, u_{i-1} \in O_{K_{i}}^{\times}=U_{K_{i}}$ and $x \in K_{i}^{\times}$, where $\bar{u}_{1}, \cdots, \bar{u}_{i-1} \in K_{i-1}$ are defined by reduction modulo $\mathfrak{p}_{K_{i}}$ of the elements $u_{1}, \cdots, u_{i-1}$ in $K_{i}$, for each $i=1,2, \cdots, n$. Therefore, there exists a surjective homomorphism called the (topological $K$-theoretic) valuation map

$$
\nu_{\mathrm{K}_{n}^{\mathrm{top}}(K)}: \mathrm{K}_{n}^{\mathrm{top}}(K) \rightarrow \mathbb{Z}
$$

on $\mathrm{K}_{n}^{\mathrm{top}}(K)$ defined by the composition

$$
\nu_{\mathrm{K}_{n}^{\mathrm{top}}(K)}: \mathrm{K}_{n}^{\mathrm{top}}\left(K_{n}\right) \stackrel{\left(\partial_{n-1}^{n}\right)^{\mathrm{top}}}{\longrightarrow} \mathrm{K}_{n-1}^{\mathrm{top}}\left(K_{n-1}\right) \stackrel{\left(\partial_{n-2}^{n-1}\right)^{\mathrm{top}}}{\longrightarrow} \cdots \stackrel{\left(\partial_{0}^{1}\right)^{\mathrm{top}}}{\longrightarrow} \mathrm{K}_{0}^{\mathrm{top}}\left(K_{0}\right)=\mathbb{Z} .
$$

Clearly, the valuation $\nu_{\mathrm{K}_{n}^{\mathrm{Milnor}}(K)}: \mathrm{K}_{n}^{\mathrm{Milnor}}(K) \rightarrow \mathbb{Z}$ factors through

as the diagram

$$
\nu_{\mathrm{K}_{n}^{\text {Milnor }}(K)}: \mathrm{K}_{n}^{\text {Milnor }}(K) \stackrel{\operatorname{red}_{\mathrm{K}_{n}^{\text {Milnor }}(K)}}{\longrightarrow} \mathrm{K}_{n}^{\text {top }}(K) \stackrel{\nu_{\mathrm{K}_{n}^{\text {top }}(K)}}{\longrightarrow} \mathbb{Z}
$$

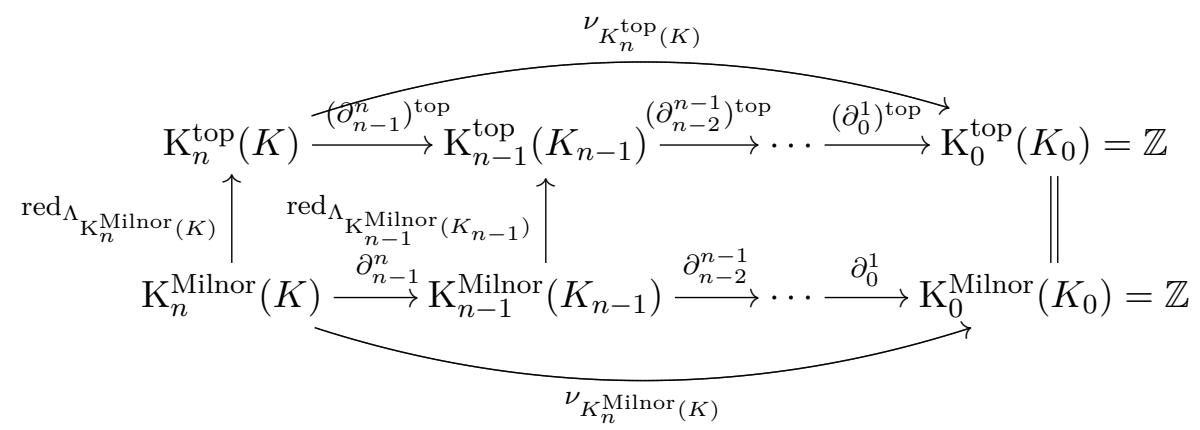

is commutative. An element $\Pi_{\mathrm{K}_{n}^{\mathrm{top}}(K)}$ of $\mathrm{K}_{n}^{\mathrm{top}}(K)$ is called a prime element of $\mathrm{K}_{n}^{\mathrm{top}}(K)$ if

$$
\nu_{\mathrm{K}_{n}^{\mathrm{top}}(K)}\left(\Pi_{\mathrm{K}_{n}^{\mathrm{top}}(K)}\right)=1 .
$$


Note that, a prime element $\Pi_{\mathrm{K}_{n}^{\mathrm{top}}(K)}$ of $\mathrm{K}_{n}^{\mathrm{top}}(K)$ can be expressed as

$$
\Pi_{\mathrm{K}_{n}^{\mathrm{top}}(K)}=\left\{t_{1, K}, \cdots, t_{n, K}\right\}^{\text {top }}+\varepsilon,
$$

where $\Pi_{K}=\left(t_{1, K}, \cdots, t_{n, K}\right)$ is a system of local parameters of $K$ and the element $\varepsilon$ lies in $\operatorname{Ker}\left(\nu_{\mathrm{K}_{n}^{\mathrm{top}}(K)}\right)$.

Let $L$ be a finite extension of the $n$-dimensional local field $K$. As $\mathrm{N}_{L / K}^{\mathrm{Milnor}}\left(\Lambda_{\mathrm{K}_{m}^{\mathrm{Milnor}}(L)}\right) \subseteq$ $\Lambda_{\mathrm{K}_{m}^{\mathrm{Milnor}}(K)}$, the norm map

$$
\mathrm{N}_{L / K}^{\mathrm{Milnor}}: \mathrm{K}_{m}^{\mathrm{Milnor}}(L) \rightarrow \mathrm{K}_{m}^{\mathrm{Milnor}}(K)
$$

induces a unique homomorphism

$$
\mathrm{N}_{L / K}^{\mathrm{top}}: \mathrm{K}_{m}^{\mathrm{top}}(L) \rightarrow \mathrm{K}_{m}^{\mathrm{top}}(K)
$$

satisfying, following [38],

$$
\left.\mathrm{N}_{L / K}^{\text {top }} \text { (open subgroup of } \mathrm{K}_{m}^{\mathrm{top}}(L)\right) \subseteq \text { open subgroup of } \mathrm{K}_{m}^{\mathrm{top}}(K)
$$

with the usual transitivity property; namely, $\mathrm{N}_{L / K}^{\text {top }}=\mathrm{N}_{M / K}^{\text {top }} \circ \mathrm{N}_{L / M}^{\text {top }}$ for every chain $K \subseteq$ $M \subseteq L$ of finite extensions of $K$. Moreover,

- The composition

$$
\mathrm{K}_{m}^{\mathrm{top}}(K) \stackrel{j_{L / K}^{\mathrm{top}}}{\longrightarrow} \mathrm{K}_{m}^{\mathrm{top}}(L) \stackrel{\mathrm{N}_{L / K}^{\mathrm{top}}}{\longrightarrow} \mathrm{K}_{m}^{\mathrm{top}}(K)
$$

is the multiplication by $[L: K]$ mapping;

- If $\sigma \in \operatorname{Aut}_{K}(L)$, then

$$
\mathrm{N}_{L / K}^{\mathrm{top}} \circ \mathrm{K}_{m}^{\mathrm{top}}(\sigma)=\mathrm{N}_{L / K}^{\mathrm{top}},
$$

where $\mathrm{K}_{m}^{\mathrm{top}}(\sigma): \mathrm{K}_{m}^{\mathrm{top}}(L) \rightarrow \mathrm{K}_{m}^{\mathrm{top}}(L)$ is the homomorphism induced by the $K$ automorphism $\sigma: L \rightarrow L$.

For any finite extension $L$ of $K$, the topological $K$-theoretic valuation map

$$
\nu_{\mathrm{K}_{n}^{\mathrm{top}}(L)}: \mathrm{K}_{n}^{\mathrm{top}}(L) \rightarrow \mathbb{Z}
$$

on $\mathrm{K}_{n}^{\mathrm{top}}(L)$ satisfies

$$
\nu_{\mathrm{K}_{n}^{\mathrm{top}}(L)}=\frac{1}{f(L / K)} \nu_{\mathrm{K}_{n}^{\mathrm{top}}(K)} \circ \mathrm{N}_{L / K}^{\mathrm{top}},
$$

where $f(L / K)=\left[L_{0}: K_{0}\right]$, because the diagram

$$
\begin{aligned}
& \mathrm{K}_{n}^{\mathrm{top}}(L) \stackrel{\left(\partial_{n-1}^{n}\right)^{\mathrm{top}}}{\longrightarrow} \mathrm{K}_{n-1}^{\mathrm{top}}\left(L_{n-1}\right) \\
& \mathrm{N}_{L / K}^{\mathrm{top}} \downarrow \\
& \mathrm{K}_{n}^{\mathrm{top}}(K) \stackrel{\left(\partial_{n-1}^{n}\right)^{\mathrm{top}}}{\longrightarrow} \mathrm{K}_{n-1}^{\mathrm{top}}\left(\mathrm{N}_{L_{n-1} / K_{n-1}}^{\mathrm{top}}\right)
\end{aligned}
$$

is commutative.

Note that, the commutative diagram (5.5) naturally induces a homomorphism

$$
\left(\partial_{n-1}^{n}\right)_{*}^{\text {top }}: \mathrm{K}_{n}^{\mathrm{top}}(K) / \mathrm{N}_{L / K}^{\mathrm{top}}\left(\mathrm{K}_{n}^{\mathrm{top}}(L)\right) \rightarrow \mathrm{K}_{n-1}^{\mathrm{top}}\left(K_{n-1}\right) / \mathrm{N}_{L_{n-1} / K_{n-1}}^{\mathrm{top}}\left(\mathrm{K}_{n-1}^{\mathrm{top}}\left(L_{n-1}\right)\right)
$$

for every finite extension $L$ of $K$.

The system of local parameters $\Pi_{K}=\left(t_{1, K}, \cdots, t_{n, K}\right) \in K^{n}$ of $K$ determines a rank $n$ discrete valuation

$$
\bar{v}_{K}: K \rightarrow \mathbb{Z}^{n} \cup\{\infty\}
$$


of $K$, which naturally determines a collection $\left\{U_{\bar{v}_{K}}^{\left(i_{\ell}, \cdots, i_{n}\right)} \mathrm{K}_{m}^{\mathrm{top}}(K)\right\}_{\left(i_{\ell}, \cdots, i_{n}\right)}$ of subgroups $U_{\bar{v}_{K}}^{\left(i_{\ell}, \cdots, i_{n}\right)} \mathrm{K}_{m}^{\mathrm{top}}(K)$ of $\mathrm{K}_{m}^{\mathrm{top}}(K)$, where $U_{\bar{v}_{K}}^{\left(i_{\ell}, \cdots, i_{n}\right)} \mathrm{K}_{m}^{\mathrm{top}}(K)$ is defined by the image

$$
\operatorname{red}_{\Lambda_{\mathrm{K}_{m}^{\mathrm{Milnor}}(K)}}: U_{\bar{v}_{K}}^{\left(i_{\ell}, \cdots, i_{n}\right)} \mathrm{K}_{m}^{\mathrm{Milnor}}(K) \mapsto \operatorname{red}_{\Lambda_{\mathrm{K}_{m}^{\mathrm{Milnor}}(K)}}\left(U_{\bar{v}_{K}}^{\left(i_{\ell}, \cdots, i_{n}\right)} \mathrm{K}_{m}^{\mathrm{Milnor}}(K)\right)
$$

in $\mathrm{K}_{m}^{\mathrm{top}}(K)$ of $U_{\bar{v}_{K}}^{\left(i_{\ell}, \cdots, i_{n}\right)} \mathrm{K}_{m}^{\mathrm{Milnor}}(K)$ under the natural homomorphism

$$
\operatorname{red}_{\Lambda_{\mathrm{K}_{m}^{\mathrm{Milnor}}(K)}}: \mathrm{K}_{m}^{\mathrm{Milnor}}(K) \rightarrow \mathrm{K}_{m}^{\mathrm{top}}(K),
$$

where $\left(i_{\ell}, \cdots, i_{n}\right) \in \mathbb{Z}^{n-\ell+1}$ satisfies $\left(i_{\ell}, \cdots, i_{n}\right) \succeq \overbrace{(0, \cdots, 0)}^{(n-\ell+1) \text {-tuple }}$, for each $\ell$ satisfying $1 \leq \ell \leq n$. The collection $\left\{U_{\bar{v}_{K}}^{\left(i_{\ell}, \cdots, i_{n}\right)} \mathrm{K}_{m}^{\mathrm{top}}(K)\right\}_{\left(i_{\ell}, \cdots, i_{n}\right)}$ is a neighborhood basis of the identity element of $\mathrm{K}_{m}^{\mathrm{top}}(K)$.

In particular, in case $\ell=n$, the group $U_{\bar{v}_{K}}^{\left(i_{n}\right)} \mathrm{K}_{m}^{\mathrm{top}}(K)$ is denoted by $U_{K_{n}}^{i_{n}} \mathrm{~K}_{m}^{\mathrm{top}}(K)$ for each $i_{n} \in \mathbb{Z}$ satisfying $i_{n} \geq 0$. Moreover,

- if $i_{n}=0$, the group $U_{\bar{v}_{K}}^{\left(i_{n}=0\right)} \mathrm{K}_{m}^{\mathrm{top}}(K)$ is denoted by $U_{K_{n}} \mathrm{~K}_{m}^{\mathrm{top}}(K)$;

- if $i_{n}=1$, the group $U_{\bar{v}_{K}}^{\left(i_{n}=1\right)} \mathrm{K}_{m}^{\text {top }}(K)$ is denoted by $V_{K_{n}} \mathrm{~K}_{m}^{\text {top }}(K)$.

In case $L$ is an algebraic extension of $K$ and $\bar{w}_{L}$ is the unique extension of the rank $n$ discrete valuation $\bar{v}_{K}$ of $K$ to $L$, the subgroup $U_{\bar{w}_{L}}^{\left(i_{\ell}, \cdots, i_{n}\right)} \mathrm{K}_{m}^{\mathrm{top}}(L)$ of $\mathrm{K}_{m}^{\mathrm{top}}(L)$, which is denoted by $U_{\bar{v}_{K}}^{\left(i_{\ell}, \cdots, i_{n}\right)} \mathrm{K}_{m}^{\mathrm{top}}(L)$, is defined by the image $\operatorname{red}_{\Lambda_{\mathrm{K}_{m}^{\mathrm{Milnor}}(L)}}\left(U_{\bar{v}_{K}}^{\left(i_{\ell}, \cdots, i_{n}\right)} \mathrm{K}_{m}^{\mathrm{Milnor}}(L)\right)$ in $\mathrm{K}_{m}^{\text {top }}(L)$ of $U_{\bar{v}_{K}}^{\left(i_{\ell}, \cdots, i_{n}\right)} \mathrm{K}_{m}^{\text {Milnor }}(L)$ under the natural homomorphism

$$
\operatorname{red}_{\Lambda_{\mathrm{K}_{m}^{\text {Milnor }}(L)}}: \mathrm{K}_{m}^{\mathrm{Milnor}}(L) \rightarrow \mathrm{K}_{m}^{\mathrm{top}}(L),
$$

where $\left(i_{\ell}, \cdots, i_{n}\right) \in \mathbb{Z}^{n-\ell+1}$ satisfies $\left(i_{\ell}, \cdots, i_{n}\right) \succeq \overbrace{(0, \cdots, 0)}^{(n-\ell+1) \text {-tuple }}$, for each $\ell$ satisfying $1 \leq \ell \leq n$.

In particular, in case $\ell=n$, the group $U_{\bar{v}_{K}}^{\left(i_{n}\right)} \mathrm{K}_{m}^{\text {top }}(L)$ is denoted by $U_{K_{n}}^{i_{n}} \mathrm{~K}_{m}^{\text {top }}(L)$ for each $i_{n} \in \mathbb{Z}$ satisfying $i_{n} \geq 0$. Moreover,

- if $i_{n}=0$, the group $U_{\bar{v}_{K}}^{\left(i_{n}=0\right)} \mathrm{K}_{m}^{\mathrm{top}}(L)$ is denoted by $U_{K_{n}} \mathrm{~K}_{m}^{\mathrm{top}}(L)$.

- if $i_{n}=1$, the group $U_{\bar{v}_{K}}^{\left(i_{n}=1\right)} \mathrm{K}_{m}^{\mathrm{top}}(L)$ is denoted by $V_{K_{n}} \mathrm{~K}_{m}^{\mathrm{top}}(L)$.

The structure of $\mathrm{K}_{n}^{\mathrm{top}}(K)$ is well-known (look at $\left.[12,14]\right)$. In fact,

$$
\mathrm{K}_{n}^{\text {top }}(K) \stackrel{\sim}{\rightarrow} \mathbb{Z}_{p} \oplus V_{K_{n}} \mathrm{~K}_{n}^{\text {top }}(K)
$$

where, as introduced above, $V_{K_{n}} \mathrm{~K}_{n}^{\text {top }}(K)$ is the image of $V_{K_{n}} \mathrm{~K}_{n}^{\text {Milnor }}(K)$ under $\operatorname{red}_{\Lambda_{\mathrm{K}_{n}^{\text {Milnor }}(K)}}$. Now, introduce the subset $\mathbb{I}_{p, n}$ of $\mathbb{Z}^{n}$ by

$$
\mathbb{I}_{p, n}=\left\{\boldsymbol{a}=\left(a_{1}, \cdots, a_{n}\right) \in \mathbb{Z}^{n}: \boldsymbol{a} \notin(p \mathbb{Z})^{n}, \mathbf{0} \prec \boldsymbol{a}\right\} .
$$

For each $\boldsymbol{a} \in \mathbb{I}_{p, n}$, consider the integer $1 \leq i(\boldsymbol{a}) \leq n$ defined uniquely by the conditions :

$$
\begin{aligned}
& -a_{i(\boldsymbol{a})+1} \equiv \cdots \equiv a_{n} \equiv 0(\bmod p) ; \\
& -a_{i(\boldsymbol{a})} \not \equiv 0(\bmod p) .
\end{aligned}
$$

Let $\theta_{1}, \cdots, \theta_{s}$ be an $\mathbb{F}_{p}$-basis of the last residue field $K_{0}=\mathbb{F}_{q}$, where $q=p^{s}$. Now, for each $\boldsymbol{a} \in \mathbb{I}_{p, n}$ and $1 \leq j \leq s$, introduce the topological Steinberg symbol $\varepsilon_{j, \boldsymbol{a}}$ in $\mathrm{K}_{n}^{\text {top }}(K)$ by

$$
\varepsilon_{j, \boldsymbol{a}}:=\left\{1+\theta_{j} \underline{t}_{K}^{\boldsymbol{a}}, t_{1, K}, \cdots, t_{i(\boldsymbol{a})-1, K}, t_{i(\boldsymbol{a})+1, K}, \cdots, t_{n, K}\right\}^{\text {top }},
$$


where $\left(t_{1, K}, \cdots, t_{n, K}\right) \in K^{n}$ is a system of local parameters of the $n$-dimensional local field $K$, and $\underline{t}_{K}^{a}:=t_{1, K}^{a_{1}} \cdots t_{n, K}^{a_{n}}$. Then, the collection $\left\{\varepsilon_{j, \boldsymbol{a}}\right\}_{\substack{1 \leq j \leq s \\ \boldsymbol{a} \in \mathbb{I}_{p, n}}}$ is a system of free topological generators of $V_{K_{n}} \mathrm{~K}_{n}^{\mathrm{top}}(K)$. Therefore, any $\xi \in \mathrm{K}_{n}^{\mathrm{top}}(K)$ can be expressed uniquely as

$$
\xi=A_{o}\left\{t_{1, K}, \cdots, t_{n, K}\right\}^{\text {top }}+\sum_{\substack{1 \leq j \leq s \\ \boldsymbol{b} \in \mathbb{I}_{p, n}}} A_{j, \boldsymbol{b}} \varepsilon_{j, \boldsymbol{b}},
$$

where $A_{o}, A_{j, \boldsymbol{b}} \in \mathbb{Z}_{p}$ for every $1 \leq j \leq s, \boldsymbol{b} \in \mathbb{I}_{p, n}$.

For more details about topological Milnor $K$-groups, look at $[12,14]$.

\section{Ramification theory of $n$-dimensional local fields}

If $K$ is a non-archimedean (=1-dimensional) local field, then there exists a very solid theory, the ramification theory of the non-archimedean local field $K$ [15]. Namely, for a finite Galois extension $L / K$ with Galois group $\operatorname{Gal}(L / K)=G$, there exists a lower filtration $\left(G_{i}\right)_{i \in \mathbb{R}_{\geq-1}}$ of $G$ defined by higher ramification subgroups $G_{i}:=\left\{\gamma \in G \mid \nu_{L}(\gamma(x)-x) \geq\right.$ $\left.i+1, \forall x \in O_{L}\right\}$ of $G$ in lower numbering, for $i \in \mathbb{R}_{\geq-1}$. The lower filtration $\left(G_{i}\right)_{i \in \mathbb{R}_{\geq-1}}$ of $G$ behaves well with respect to "passing to the subgroups" in the sense that, for any subgroup $H$ of $G, H_{i}=H \cap G_{i}$, for every $i \in \mathbb{R}_{\geq-1}$. On the other hand, the lower filtration $\left(G_{i}\right)_{i \in \mathbb{R}_{\geq-1}}$ of $G$ does not behave well with respect to "taking quotients". That is, there exists $H$ a normal subgroup of $G$ such that $(G / H)_{i} \neq G_{i} H / H$ for some $i \in \mathbb{R}_{\geq-1}$. In fact, defining $G^{j}=G_{\psi_{L / K}(j)}$, for all $j \in \mathbb{R}_{\geq-1}$, where $\psi_{L / K}: \mathbb{R}_{\geq-1} \rightarrow \mathbb{R}_{\geq-1}$ the Hasse-Herbrand function of the extension $L / K$ is the piecewise linear increasing function with inverse $\psi_{L / K}^{-1}=\phi_{L / K}: \mathbb{R}_{\geq-1} \rightarrow \mathbb{R}_{\geq-1}$ defined by $\phi_{L / K}(i)=\int_{0}^{i} \frac{d t}{\left[G_{0}: G_{t}\right]}$ for $i \in \mathbb{R}_{\geq-1}$ produces the upper filtration $\left(G^{j}\right)_{j \in \mathbb{R}_{\geq-1}}$ of $G$ defined by higher ramification subgroups $G^{j}$ of $G$ in upper numbering, for $j \in \mathbb{R}_{\geq-1}$, which behaves well with respect to "taking quotients" now. That is, for any normal subgroup $H$ of $G,(G / H)^{j}=G^{j} H / H$ for every $j \in \mathbb{R}_{\geq-1}$. Thus, higher ramification subgroups $G^{j}$ of $G$ in upper numbering, for $j \in \mathbb{R}_{\geq-1}$, can be used to define higher ramification subgroups $G_{K}^{j}$ of the absolute Galois group $G_{K}$ in upper numbering, for $j \in \mathbb{R}_{\geq-1}$.

If the finite extension $L / K$ is furthermore assumed to be abelian, the most important property of the upper filtration $\left(G^{j}\right)_{j \in \mathbb{R}_{\geq-1}}$ of $G$ is that the local abelian Hasse reciprocity law

$$
\operatorname{Rec}_{L / K_{*}}: K^{\times} / \mathrm{N}_{L / K}\left(L^{\times}\right) \stackrel{\sim}{\rightarrow} G
$$

of the abelian extension $L / K$ maps the subgroup $U_{K}^{j} /\left(U_{K}^{j} \cap \mathrm{N}_{L / K}\left(L^{\times}\right)\right)$of $K^{\times} / \mathrm{N}_{L / K}\left(L^{\times}\right)$ to the higher ramification subgroup $G^{j}$ of $G$ in upper numbering for every $j \in \mathbb{R}_{\geq-1}$. Note that, both filtrations $\left(U_{K}^{j}\right)_{j \in \mathbb{R}_{\geq-1}}$ and $\left(G^{j}\right)_{j \in \mathbb{R}_{\geq-1}}$ form bases of neighbourhoods of $K^{\times}$and of $G$ respectively.

Therefore, in principle, we should be able to define an upper ramification theory on a "valued field" $K$ in the situations where some class field theory for the valued field $K$ is available. For instance, using this principle, Lomadze [35] initiated the ramification theory of abelian extensions of 2-dimensional local fields of characteristic $p>0$ by defining an upper filtration on corresponding abelian Galois groups using local abelian 2-dimensional class field theory, which is the subject of Section 7. On the other hand, if $K$ is an $n$-dimensional local field with $n \geq 2$, we observe that there are two different, yet not totally unrelated, valuations on $K$. Namely, there exists a rank $n$ discrete valuation $\bar{v}_{K}: K \rightarrow \mathbb{Z}^{n} \cup\{\infty\}$ defined on $K$, and also a discrete valuation $\nu_{K_{n}}: K_{n} \rightarrow \mathbb{Z} \cup\{\infty\}$ defined on $K_{n}=K$. So, there are two "seemingly different" valued field structures on $K$. Therefore, it is natural to expect different types of ramification theories on $K$, which are:

- Zhukov type ramification theory on $K[49,51]$, which generalizes [23, 35];

- Abbes-Saito type ramification theory on $K$ [1,49], which generalizes [19,24]. 
In what follows, we shall choose Abbes-Saito type ramification theory on the $n$-dimensional local field $K$. In fact, in Abbes-Saito theory on $K$, there are two filtrations $G_{K, \text { nlog }}^{\bullet}$ and $G_{K, \log }^{\bullet}$ on the absolute Galois group $G_{K}$ of $K$ both indexed by the set of non-negative rational numbers $\mathbb{Q}_{\geq 0}$, called the upper non-logarithmic ramification filtration of $G_{K}$ and the upper logarithmic ramification filtration of $G_{K}$, respectively [49, Subsection 6.1]. Moreover, specializing only to abelian extensions of $K$, Abbes-Saito non-logarithmic ramification theory of abelian extensions of $K$ coincides with the ramification theory of Kato, which is defined only for abelian extensions of $K$ [28] and which also behaves well with respect to the existing local abelian Kato-Parshin reciprocity law of $K^{* *}$ [24], the main subject of this review.

The ramification theory of Kato on the $n$-dimensional local field $K$, which is modelled after the work of Hyodo [19], first constructs a conductor $\operatorname{KSw}(\chi)$ for $\chi \in H^{1}(K)=$ $\operatorname{Hom}\left(G_{K}^{\mathrm{ab}}, \mathbb{Q} / \mathbb{Z}\right)$, called the Kato-Swan conductor for a 1-dimensional representation $\chi$ : $G_{K}^{\mathrm{ab}} \rightarrow \mathbb{Q} / \mathbb{Z}$ of $G_{K}^{\mathrm{ab}}$, where $K$ is a complete discrete valuation field with any residue field $\kappa_{K}$. The conductor $\operatorname{KSw}(\chi)$ for the 1-dimensional representation $\chi: G_{K}^{\mathrm{ab}} \rightarrow \mathbb{Q} / \mathbb{Z}$ of $G_{K}^{\mathrm{ab}}$ is characterized by the smallest integer $f \geq 0$ satisfying

$$
U_{K}^{f+1} \subseteq N_{L_{\chi} / K} L_{\chi}^{\times},
$$

where $L_{\chi} / K$ is the subextension of $K^{\mathrm{ab}} / K$ fixed by $\chi: G_{K}^{\mathrm{ab}} \rightarrow \mathbb{Q} / \mathbb{Z}$. So, there exists an upper filtration $G_{K}^{\mathrm{ab}, \bullet}$ on $G_{K}^{\mathrm{ab}}$, called the Kato filtration on $G_{K}^{\mathrm{ab}}$, satisfying

$$
\operatorname{KSw}(\chi)=\inf \left\{a>0 \mid G_{K}^{\mathrm{ab}, a} \subseteq \operatorname{Ker}(\chi)\right\},
$$

for any $\chi: G_{K}^{\mathrm{ab}} \rightarrow \mathbb{Q} / \mathbb{Z}$.

\section{Local abelian $K$-theoretic class field theory of Kato-Parshin}

Fix a separable closure $K^{\text {sep }}$ of the $n$-dimensional local field $K$ and let $K^{\text {ab }} \subset K^{\text {sep }}$ be the maximal abelian extension of $K$ inside $K^{\text {sep }}$.

The profinite completion $\widehat{\mathrm{K}}_{n}^{\text {top }}(K)$ of $\mathrm{K}_{n}^{\text {top }}(K)$ with respect to the norm map is defined by the projective limit

$$
\widehat{\mathrm{K}}_{n}^{\mathrm{top}}(K):={\underset{E}{E}}_{\lim _{n}} \mathrm{~K}_{n}^{\mathrm{top}}(K) / \mathrm{N}_{E / K}^{\mathrm{top}}\left(\mathrm{K}_{n}^{\mathrm{top}}(E)\right),
$$

where $E$ runs over all finite extensions of the $n$-dimensional local field $K$ inside $K^{\text {ab }}$, with respect to the connecting morphisms

$$
\mathrm{K}_{n}^{\mathrm{top}}(K) / \mathrm{N}_{E / K}^{\mathrm{top}}\left(\mathrm{K}_{n}^{\mathrm{top}}(E)\right) \stackrel{c_{E}^{E^{\prime}}}{\longleftarrow} \mathrm{K}_{n}^{\mathrm{top}}(K) / \mathrm{N}_{E^{\prime} / K}^{\mathrm{top}}\left(\mathrm{K}_{n}^{\mathrm{top}}\left(E^{\prime}\right)\right)
$$

defined for any two finite extensions $E$ and $E^{\prime}$ of $K$ inside $K^{\text {ab }}$ satisfying $E \subseteq E^{\prime}$ by

$$
\alpha \quad\left(\bmod \mathrm{N}_{E / K}^{\mathrm{top}}\left(\mathrm{K}_{n}^{\mathrm{top}}(E)\right)\right) \stackrel{c_{E}^{E^{\prime}}}{\longleftarrow} \alpha \quad\left(\bmod \mathrm{N}_{E^{\prime} / K}^{\mathrm{top}}\left(\mathrm{K}_{n}^{\mathrm{top}}\left(E^{\prime}\right)\right)\right)
$$

for every $\alpha \in \mathrm{K}_{n}^{\mathrm{top}}(K)$.

Given any finite extension $L$ of $K$, then the homomorphism $\mathrm{N}_{L / K}^{\mathrm{top}}: \mathrm{K}_{n}^{\mathrm{top}}(L) \rightarrow \mathrm{K}_{n}^{\mathrm{top}}(K)$ extends to profinite completions, and defines a continuous homomorphism

$$
\widehat{\mathrm{N}}_{L / K}^{\mathrm{top}}: \widehat{\mathrm{K}}_{n}^{\mathrm{top}}(L) \rightarrow \widehat{\mathrm{K}}_{n}^{\mathrm{top}}(K)
$$

\footnotetext{
${ }^{* *}$ So, it is natural to expect that Abbes-Saito type non-logarithmic ramification theory on the $n$-dimensional local field $K$ behaves well with respect to the "hypothetical" local non-abelian Kato-Parshin reciprocity law of $K$, which still needs construction [20].
} 
satisfying the transitivity condition, as the diagram

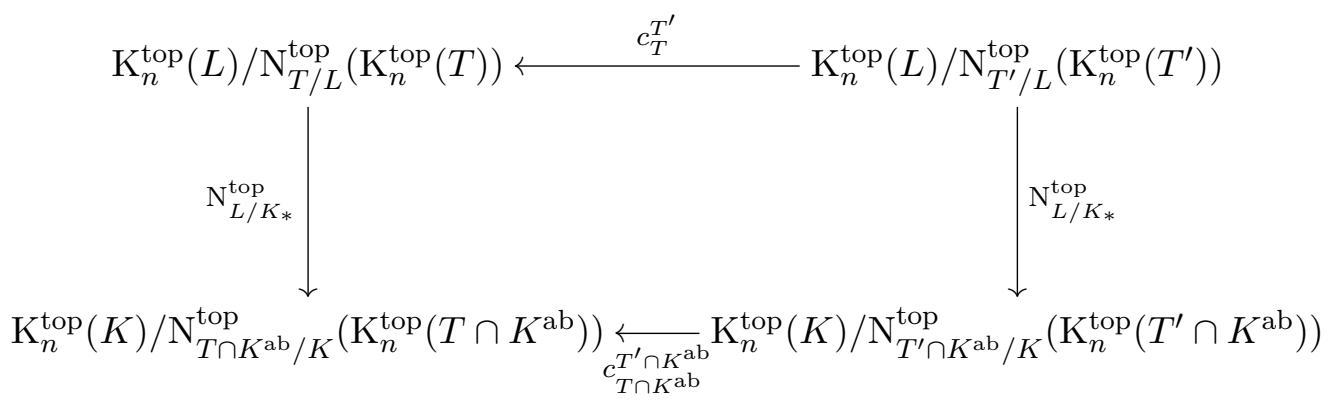

is commutative, where the vertical arrows $\mathrm{N}_{L / K_{*}}^{\text {top }}$ are the induced morphisms from $\mathrm{N}_{L / K}^{\text {top }}$, for each finite extension $T$ and $T^{\prime}$ of $L$ inside $L^{\mathrm{ab}}$ satisfying $T \subseteq T^{\prime}$.

Recall that, local abelian $n$-dimensional $K$-theoretic class field theory for $K$ establishes a unique natural algebraic and topological [38] isomorphism

$$
\operatorname{Rec}_{K}: \widehat{\mathrm{K}}_{n}^{\mathrm{top}}(K) \stackrel{\sim}{\rightarrow} G_{K}^{\mathrm{ab}},
$$

called the local abelian $n$-dimensional Kato-Parshin reciprocity law of $K$, which, among other things, has the following properties :

(1) For every abelian extension $L / K$, the surjective homomorphism

$$
\operatorname{Rec}_{L / K}: \widehat{\mathrm{K}}_{n}^{\mathrm{top}}(K) \stackrel{\operatorname{Rec}_{K}}{\longrightarrow} G_{K}^{\mathrm{ab}} \stackrel{\operatorname{res}_{L}}{\longrightarrow} \operatorname{Gal}(L / K)
$$

has kernel

$$
\operatorname{Ker}\left(\operatorname{Rec}_{L / K}\right)=\widehat{\mathrm{N}}_{L / K}^{\mathrm{top}}\left(\widehat{\mathrm{K}}_{n}^{\mathrm{top}}(L)\right)=\bigcap_{K} \underset{\text { finite }}{\subseteq}{ }_{\mathcal{C}} \widehat{\mathrm{N}}_{F / K}^{\mathrm{top}}\left(\widehat{\mathrm{K}}_{n}^{\mathrm{top}}(F)\right)=: \mathfrak{N}_{L / K}^{\mathrm{top}},
$$

and induces a topological group isomorphism

$$
\operatorname{Rec}_{L / K_{*}}: \widehat{\mathrm{K}}_{n}^{\mathrm{top}}(K) / \mathfrak{N}_{L / K}^{\mathrm{top}} \stackrel{\sim}{\rightarrow} \operatorname{Gal}(L / K)
$$

called the local abelian $n$-dimensional Kato-Parshin reciprocity law of $L / K$;

(2) (Existence theorem). For each abelian extension $L / K$, the mapping

$$
L / K \mapsto \mathfrak{N}_{L / K}^{\text {top }}
$$

defines a bijective correspondence

$$
\{L / K: \text { abelian }\} \rightleftarrows\left\{\mathfrak{N}: \mathfrak{N} \underset{\text { "closed" }}{\leq} \widehat{\mathrm{K}}_{n}^{\text {top }}(K)\right\} .
$$

For Kato's approach to the existence theorem, look at [25];

(3) (Functoriality). For any finite extension $L / K$,

$$
\left.\operatorname{Rec}_{L}(x)\right|_{K^{\mathrm{ab}}}=\operatorname{Rec}_{K}\left(\widehat{\mathrm{N}}_{L / K}^{\mathrm{top}}(x)\right),
$$

for every $x \in \widehat{\mathrm{K}}_{n}^{\mathrm{top}}(L)$, and

$$
\operatorname{Rec}_{L}\left(\widehat{j_{L / K}^{\text {top }}}(x)\right)=V_{L / K}\left(\operatorname{Rec}_{K}(x)\right),
$$

for every $x \in \widehat{\mathrm{K}}_{n}^{\mathrm{top}}(K)$. That is, the following squares

$$
\begin{aligned}
& \widehat{\mathrm{K}}_{n}^{\mathrm{top}}(L) \stackrel{\operatorname{Rec}_{L}}{\longrightarrow} G_{L}^{\mathrm{ab}} \\
& \widehat{\mathrm{K}}_{n}^{\mathrm{top}}(L) \stackrel{\operatorname{Rec}_{L}}{\longrightarrow} G_{L}^{\mathrm{ab}} \\
& \widehat{\mathrm{N}}_{L / K}^{\text {top }} \downarrow \downarrow \text { res }_{K^{\mathrm{ab}}} \\
& \widehat{\mathrm{K}}_{n}^{\mathrm{top}}(K) \stackrel{\operatorname{Rec}_{K}}{\longrightarrow} G_{K}^{\mathrm{ab}} \\
& \widehat{j_{L / K}^{\text {top }}} \uparrow \quad \uparrow V_{L / K}: \text { Verlagerung } \\
& \widehat{\mathrm{K}}_{n}^{\mathrm{top}}(K) \stackrel{\operatorname{Rec}_{K}}{\longrightarrow} G_{K}^{\mathrm{ab}}
\end{aligned}
$$

are commutative; 
(4) The square

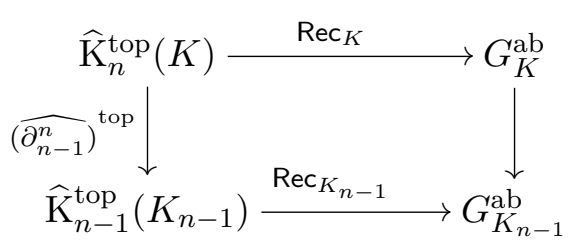

is commutative, where the left-vertical arrow

$$
{\widehat{\left(\partial_{n-1}^{n}\right)}}^{\text {top }}: \widehat{\mathrm{K}}_{n}^{\mathrm{top}}(K) \rightarrow \widehat{\mathrm{K}}_{n-1}^{\mathrm{top}}\left(K_{n-1}\right)
$$

is defined by the commutativity of the diagram

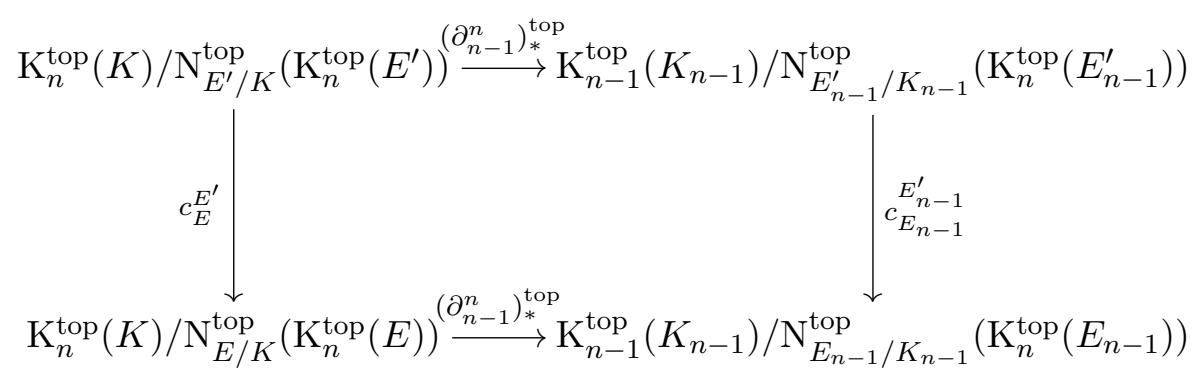

where $E$ and $E^{\prime}$ are finite extensions of $K$ inside $K^{\text {ab }}$ satisfying $E \subseteq E^{\prime}$;

(5) (Ramification theoretic properties) Let $\chi \in H^{1}(K)$; that is, let $\chi: G_{K}^{\mathrm{ab}} \rightarrow \mathbb{Q} / \mathbb{Z}$ be a character of $G_{K}^{\mathrm{ab}}$, and let $L_{\chi}$ be the finite extension of $K$ in $K^{\mathrm{ab}}$ such that $\operatorname{Ker}(\chi)=$ $\operatorname{Gal}\left(K^{\mathrm{ab}} / L_{\chi}\right)$. The Kato-Swan conductor $\operatorname{KSw}(\chi)$ of the character $\chi: G_{K}^{\mathrm{ab}} \rightarrow \mathbb{Q} / \mathbb{Z}$, defined in Section 6 as the smallest integer $f \geq 0$ satisfying $U_{K}^{f+1} \subseteq N_{L_{\chi} / K} L_{\chi}^{\times}$, is furthermore the smallest integer $f \geq 0$ such that

$$
U_{\bar{v}_{K}}^{\left(i_{1}, \cdots, i_{n}\right)} \mathrm{K}_{n}^{\mathrm{top}}(K) \subseteq \mathfrak{N}_{L_{\chi} / K}^{\mathrm{top}}
$$

whenever $i_{n}>f$.

For details about local abelian $K$-theoretic class field theory, look at [7-9], [22], and $[42,44,45]$.

There are four main approaches to construct the local abelian $n$-dimensional $K$-theoretic class field theory:

- The explicit approach of Fesenko [7-9] is based on extending the local abelian Hasse reciprocity law construction of Neukirch-Iwasawa [39,40] and on extending the local norm residue symbol construction of Hazewinkel [17] to the setting of $n$-dimensional local fields;

- Kato's approach [22,25] is cohomological and extends Tate's construction of the local abelian Hasse reciprocity law [48];

- Koya on the other hand [30-32], using Lichtenbaum's complexes $\mathbb{Z}(i)$ [34], generalizes class formation approach of local abelian class field theory to construct the local abelian 2-dimensional class field theory, which is extended and streamlined by Spiess [47] to the $n$-dimensional setting;

- The final approach, due to Parshin [42,44,45], which is the genesis of the whole program, generalizes Kawada-Satake construction of local abelian class field theory [29] to construct the local abelian $n$-dimensional class field theory in positive characteristic.

In this work we shall review Fesenko's explicit approach, where as stated above, the idea is to generalize the classical Neukirch-Iwasawa and Hazewinkel methods to higherdimensional local fields, which will be recalled next with extra care following closely $[10,11$, 13]. The explicit approach also has the advantage of extending local abelian $n$-dimensional 
$K$-theoretic class field theory to the non-abelian setting; namely, constructing the local non-abelian $n$-dimensional $K$-theoretic class field theory [20].

As first recollection, Fesenko's extension of Neukirch-Iwasawa method to $n$-dimensional local fields can be very briefly summarized as follows: Let $L$ denote a finite Galois extension of the $n$-dimensional local field $K$ in a fixed $K^{\text {sep }}$. As usual, let $L^{\text {pur }}=L K^{\text {pur }}$. For any $\sigma \in \operatorname{Gal}(L / K)$, let $\widetilde{\sigma}$ be any element of $\operatorname{Gal}\left(L^{\text {pur }} / K\right)$ such that:

$$
\begin{aligned}
& -\left.\widetilde{\sigma}\right|_{L}=\sigma ; \\
& -\left.\widetilde{\sigma}\right|_{K} \text { pur }=\varphi_{K}^{i} \text { for some } i \in \mathbb{Z} .
\end{aligned}
$$

The $n$-dimensional Neukirch-Iwasawa map

$$
\mathscr{N}_{L / K}: \operatorname{Gal}(L / K) \rightarrow \mathrm{K}_{n}^{\mathrm{top}}(K) / \mathrm{N}_{L / K}^{\mathrm{top}}\left(\mathrm{K}_{n}^{\mathrm{top}}(L)\right)
$$

of $L / K$ is then defined by

$$
\mathscr{N}_{L / K}: \sigma \mapsto \mathrm{N}_{\Sigma / K}^{\text {top }}\left(\Pi_{\mathrm{K}_{n}^{\text {top }}(\Sigma)}\right) \quad\left(\bmod \mathrm{N}_{L / K}^{\text {top }}\left(\mathrm{K}_{n}^{\text {top }}(L)\right)\right),
$$

where $\Sigma$ denotes the fixed field of $\widetilde{\sigma}$ and $\Pi_{K_{n}^{\text {top }}(\Sigma)}$ any prime element of $\mathrm{K}_{n}^{\mathrm{top}}(\Sigma)$. This map does not depend on the choice of lifting $\widetilde{\sigma}$ of $\sigma$ to $L^{\text {pur }}$ and to the choice of prime element $\Pi_{\mathrm{K}_{n}^{\mathrm{top}}(\Sigma)}$ of $\mathrm{K}_{n}^{\mathrm{top}}(\Sigma)$. Moreover, the $n$-dimensional Neukirch-Iwasawa map $\mathscr{N}_{L / K}$ : $\operatorname{Gal}(L / K) \rightarrow \mathrm{K}_{n}^{\mathrm{top}}(K) / \mathrm{N}_{L / K}^{\mathrm{top}}\left(\mathrm{K}_{n}^{\mathrm{top}}(L)\right)$ of $L / K$ induces a topological group homomorphism

$$
\mathscr{N}_{L / K}^{\mathrm{ab}}: \operatorname{Gal}(L / K)^{\mathrm{ab}} \rightarrow \mathrm{K}_{n}^{\mathrm{top}}(K) / \mathrm{N}_{L / K}^{\mathrm{top}}\left(\mathrm{K}_{n}^{\mathrm{top}}(L)\right),
$$

which is actually the inverse of the local abelian $n$-dimensional reciprocity law of $L / K$.

As second recollection, Fesenko's generalization of Hazewinkel's method to $n$-dimensional local fields can be sketched as follows: First assume that the $n$-dimensional local field $K$ is of positive characteristic, which is the easier case, as the Galois descent for $\mathrm{K}_{*}^{\text {top }}$-groups holds. Let $L$ denote a finite Galois extension of $K$ in a fixed $K^{\text {sep }}$, and let $K^{\text {pur }}$ denote the maximal purely unramified extension of $K$ in $K^{\mathrm{sep}}$. Assume further that $L / K$ is linearly disjoint with $K^{\text {pur }} / K$; that is, the extension $L / K$ is totally ramified by $(2.1)$. Recall that, the $n^{\text {th }}$ topological Milnor $K$-group $\mathrm{K}_{n}^{\text {top }}\left(K^{\text {pur }}\right)$ of $K^{\text {pur }}$ is defined by the direct limit

$$
\mathrm{K}_{n}^{\mathrm{top}}\left(K^{\mathrm{pur}}\right)=\underset{K^{\prime}}{\lim } \mathrm{K}_{n}^{\mathrm{top}}\left(K^{\prime}\right),
$$

where $K^{\prime}$ runs over all finite extensions of $K$ in $K^{\text {pur }}$, with respect to the connecting morphisms

$$
j_{K^{\prime \prime} / K^{\prime}}^{\text {top }}: \mathrm{K}_{n}^{\mathrm{top}}\left(K^{\prime}\right) \rightarrow \mathrm{K}_{n}^{\mathrm{top}}\left(K^{\prime \prime}\right)
$$

defined for any two finite extensions $K^{\prime}$ and $K^{\prime \prime}$ of $K$ inside $K^{\text {pur }}$ satisfying $K^{\prime} \subseteq K^{\prime \prime}$. Introduce the group $\mathrm{K}_{n}^{\text {top }}\left(L^{\text {pur }}\right)$ similary and define a subgroup $V(L / K)$ of $\mathrm{K}_{n}^{\text {top }}\left(L^{\text {pur }}\right)$ by

$$
V(L / K)=\left\langle\sigma(\alpha)-\alpha \mid \sigma \in \operatorname{Gal}\left(L^{\text {pur }} / K^{\text {pur }}\right), \alpha \in V_{K_{n}} K_{n}^{\text {top }}\left(L^{\text {pur }}\right)\right\rangle .
$$

Then $V(L / K) \subseteq \operatorname{Ker}\left(\mathrm{N}_{L^{\text {pur }} / K^{\text {pur }}}^{\text {top }}\right)$ and the norm map $\mathrm{N}_{L^{\text {pur }} / K^{\text {pur }}}^{\text {top }}: \mathrm{K}_{n}^{\text {top }}\left(L^{\text {pur }}\right) \rightarrow \mathrm{K}_{n}^{\text {top }}\left(K^{\text {pur }}\right)$, which is surjective, induces a morphism

$$
\mathrm{N}_{L^{\text {pur }} / K_{*}^{\text {pur }} *}^{\text {top }}: \mathrm{K}_{n}^{\text {top }}\left(L^{\text {pur }}\right) / V(L / K) \rightarrow \mathrm{K}_{n}^{\text {top }}\left(K^{\text {pur }}\right)
$$

sitting in the short exact sequence

$$
1 \rightarrow \operatorname{Gal}\left(L^{\mathrm{pur}} / K^{\mathrm{pur}}\right) \stackrel{c}{\rightarrow} \mathrm{K}_{n}^{\mathrm{top}}\left(L^{\mathrm{pur}}\right) / V(L / K) \stackrel{\mathrm{N}_{L^{\mathrm{pur}} / K^{\mathrm{pur}}}^{\mathrm{top}}}{\rightarrow} \mathrm{K}_{n}^{\mathrm{top}}\left(K^{\mathrm{pur}}\right) \rightarrow 0,
$$

where the arrow

is defined by

$$
c: \operatorname{Gal}\left(L^{\mathrm{pur}} / K^{\mathrm{pur}}\right) \rightarrow \mathrm{K}_{n}^{\mathrm{top}}\left(L^{\mathrm{pur}}\right) / V(L / K)
$$

$$
c(\sigma)=\sigma\left(\Pi_{\mathrm{K}_{n}^{\mathrm{top}}\left(L^{\mathrm{pur}}\right)}\right)-\Pi_{\mathrm{K}_{n}^{\mathrm{top}}\left(L^{\mathrm{pur}}\right)} \quad(\bmod V(L / K)),
$$


for every $\sigma \in \operatorname{Gal}\left(L^{\text {pur }} / K^{\text {pur }}\right)$, which is independent of the choice of $\Pi_{K_{n}^{\text {top }}\left(L^{\text {pur }}\right)}$. Now, for $\varepsilon \in \operatorname{Ker}\left(\nu_{\mathrm{K}_{n}^{\text {top }}(K)}\right)$ there exists $\eta_{\varepsilon} \in \mathrm{K}_{n}^{\text {top }}\left(L^{\text {pur }}\right)$ such that $\varepsilon=\mathrm{N}_{L^{\text {pur }} / K^{\text {pur }}}^{\text {top }}\left(\eta_{\varepsilon}\right)$. Let $\varphi: L^{\text {pur }} \rightarrow L^{\text {pur }}$ denote a lifting of the Frobenius automorphism $\varphi_{K}: K^{\text {pur }} \rightarrow K^{\text {pur }}$ of $K^{\text {pur }}$ to $L^{\text {pur }}$. Then, $\varphi\left(\eta_{\varepsilon}\right)-\eta_{\varepsilon}(\bmod V(L / K)) \in \operatorname{Ker}\left(\mathrm{N}_{L^{\text {pur }} / K^{\text {pur }}}^{\text {to }}\right)$ and as the sequence (7.1) is exact, there exists $\widetilde{\sigma}_{\varepsilon} \in \operatorname{Gal}\left(L^{\text {pur }} / K^{\text {pur }}\right)$ so that

$$
c\left(\widetilde{\sigma}_{\varepsilon}\right)=\widetilde{\sigma}_{\varepsilon}\left(\Pi_{K_{n}^{\mathrm{top}}\left(L^{\mathrm{pur}}\right)}\right)-\Pi_{\mathrm{K}_{n}^{\mathrm{top}}\left(L^{\mathrm{pur}}\right)}(\bmod V(L / K))=\varphi\left(\eta_{\varepsilon}\right)-\eta_{\varepsilon} \quad(\bmod V(L / K)) .
$$

Then, there exists a unique and well-defined continuous homomorphism

$$
\mathscr{H}_{L / K}: \mathrm{K}_{n}^{\text {top }}(K) / \mathrm{N}_{L / K}^{\text {top }}\left(\mathrm{K}_{n}^{\text {top }}(L)\right) \rightarrow \operatorname{Gal}(L / K)^{\text {ab }}
$$

satisfying

$$
\mathscr{H}_{L / K}:\left.\varepsilon \quad\left(\bmod \mathrm{N}_{L / K}^{\mathrm{top}}\left(\mathrm{K}_{n}^{\mathrm{top}}(L)\right)\right) \mapsto \widetilde{\sigma}_{\varepsilon}^{-1}\right|_{L \cap K^{\mathrm{ab}}},
$$

for all $\varepsilon \in \operatorname{Ker}\left(\nu_{\mathrm{K}_{n}^{\mathrm{top}}(K)}\right)$, called the $n$-dimensional Hazewinkel map of $L / K$, where $L / K$ is a finite Galois extension linearly disjoint with $K^{\text {pur }} / K$.

Let $L / K$ denote a finite Galois extension which is linearly disjoint with $K^{\text {pur }} / K$, where $\operatorname{char}(K)>0$. It turns out that, the $n$-dimensional Neukirch-Iwasawa map of $L / K$ and the $n$-dimensional Hazewinkel map of $L / K$ are inverses of each other; that is,

$$
\mathscr{H}_{L / K} \circ \mathscr{N}_{L / K}^{\mathrm{ab}}=\operatorname{Id}_{\mathrm{Gal}(L / K)^{\mathrm{ab}}} \text { and } \mathscr{N}_{L / K}^{\mathrm{ab}} \circ \mathscr{H}_{L / K}=\operatorname{Id}_{\mathrm{K}_{n}^{\mathrm{top}}(K) / \mathrm{N}_{L / K}^{\mathrm{top}}\left(\mathrm{K}_{n}^{\mathrm{top}}(L)\right)} \cdot
$$

In case $\operatorname{char}(K)=0$, unfortunately the construction sketched for the positive characteristic case does not work for $p$-extensions $L$ over $K$ in general. However, there is a method to overcome this difficulty. In fact, there is a special class of $p$-extensions $L$ over $K$, called strong Artin-Schreier trees [10,11,13], where the construction outlined for char. $>0$ works perfectly well. In fact, we have the short exact sequence (7.1) for strong Artin-Schreier trees. That is, if $L / K$ is a strong Artin-Schreier tree, then the following sequence

$$
1 \rightarrow \operatorname{Gal}(L / K) \stackrel{c}{\rightarrow} V_{K_{n}} \mathrm{~K}_{n}^{\mathrm{top}}\left(L^{\mathrm{pur}}\right) / V(L / K) \stackrel{\mathrm{N}_{L^{\mathrm{pur}} / K^{\mathrm{pur}}}^{\mathrm{top}}}{\longrightarrow} V_{K_{n}} \mathrm{~K}_{n}^{\mathrm{top}}\left(K^{\mathrm{pur}}\right) \rightarrow 0,
$$

is exact. Therefore, for a finite strong Artin-Schreier tree $L / K$ linearly disjoint with $K^{\text {pur }} / K$; that is the extension $L / K$ is totally ramified by $(2.1)$, there exists a unique and well-defined continuous homomorphism

$$
\mathscr{H}_{L / K}: V_{K_{n}} \mathrm{~K}_{n}^{\mathrm{top}}(K) / \mathrm{N}_{L / K}^{\mathrm{top}}\left(V_{K_{n}} \mathrm{~K}_{n}^{\mathrm{top}}(L)\right) \rightarrow \operatorname{Gal}(L / K)^{\mathrm{ab}},
$$

the $n$-dimensional Hazewinkel map of $L / K$, constructed as in the char. $>0$ case, which further satisfies

$$
\mathscr{H}_{L / K} \circ \mathscr{N}_{L / K}^{\mathrm{ab}}=\operatorname{Id}_{\mathrm{Gal}(L / K)^{\mathrm{ab}}} .
$$

Therefore, if $L / K$ is a finite strong Artin-Schreier tree linearly disjoint with $K^{\text {pur }} / K$, then the continuous homomorphism

$$
\mathscr{H}_{L / K}: V_{K_{n}} \mathrm{~K}_{n}^{\mathrm{top}}(K) / \mathrm{N}_{L / K}^{\mathrm{top}}\left(V_{K_{n}} \mathrm{~K}_{n}^{\mathrm{top}}(L)\right) \rightarrow \operatorname{Gal}(L / K)^{\mathrm{ab}}
$$

is a surjection, and the continuous homomorphism

$$
\mathscr{N}_{L / K}^{\mathrm{ab}}: \operatorname{Gal}(L / K)^{\mathrm{ab}} \rightarrow \mathrm{K}_{n}^{\mathrm{top}}(K) / \mathrm{N}_{L / K}^{\mathrm{top}}\left(\mathrm{K}_{n}^{\mathrm{top}}(L)\right)
$$

is an injection. Now, the class of all strong Artin-Schreier trees over $K$ is "dense" in the class of all $p$-extensions of $K$ in the sense that, for any totally ramified finite Galois $p$ extension $L / K$, there exists a totally ramified finite $p$-extension $Q_{L} / K$ such that $L Q_{L} / Q_{L}$ is a strong Artin-Schreier tree and $L^{\text {pur }} \cap Q_{L}^{\text {pur }}=K^{\text {pur }}$. So let $L / K$ be a totally ramified

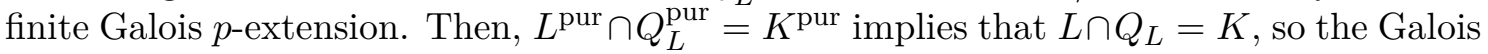
extension $L / K$ and the $p$-extension $Q_{L} / K$ are linearly disjoint. Therefore, the restriction 
map $\operatorname{Res}_{L}^{L Q_{L}}: \operatorname{Gal}\left(L Q_{L} / Q_{K}\right) \stackrel{\sim}{\rightarrow} \operatorname{Gal}(L / K)$ is an isomorphism of profinite groups, and the following square

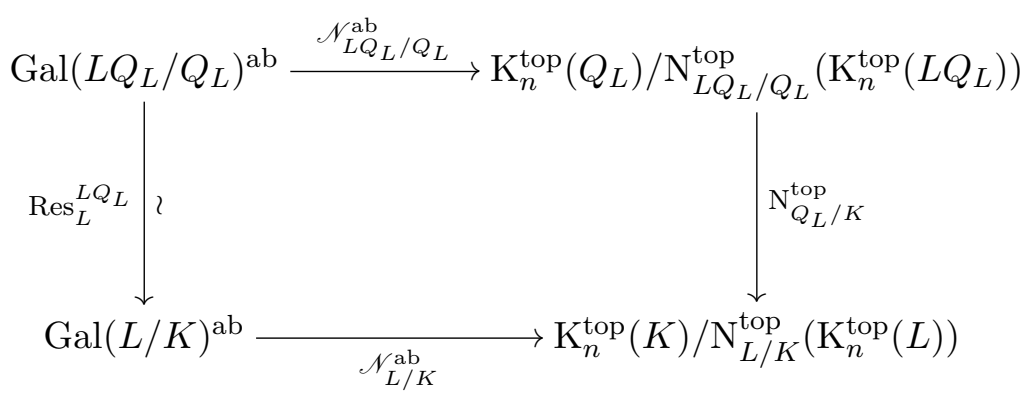

is commutative. Therefore,

$$
\mathscr{N}_{L / K}^{\mathrm{ab}}: \operatorname{Gal}(L / K)^{\mathrm{ab}} \rightarrow \mathrm{K}_{n}^{\mathrm{top}}(K) / \mathrm{N}_{L / K}^{\mathrm{top}}\left(\mathrm{K}_{n}^{\mathrm{top}}(L)\right)
$$

is an injective homomorphism of topological groups, since the Neukirch-Iwasawa map

$$
\mathscr{N}_{L Q_{L} / Q_{L}}^{\mathrm{ab}}: \operatorname{Gal}\left(L Q_{L} / Q_{L}\right)^{\mathrm{ab}} \rightarrow \mathrm{K}_{n}^{\mathrm{top}}\left(Q_{L}\right) / \mathrm{N}_{L Q_{L} / Q_{L}}^{\mathrm{top}}\left(\mathrm{K}_{n}^{\mathrm{top}}\left(L Q_{L}\right)\right)
$$

of $L Q_{L} / Q_{L}$ is an injective arrow by equality (7.3) as $L Q_{L} / Q_{L}$ is a finite strong ArtinSchreier tree linearly disjoint with $Q_{L}^{\text {pur }} / Q_{L}{ }^{\dagger \dagger}$. The surjectivity of the $n$-dimensional Neukirch-Iwasawa map

$$
\mathscr{N}_{L / K}^{\mathrm{ab}}: \operatorname{Gal}(L / K)^{\mathrm{ab}} \rightarrow \mathrm{K}_{n}^{\mathrm{top}}(K) / \mathrm{N}_{L / K}^{\mathrm{top}}\left(\mathrm{K}_{n}^{\mathrm{top}}(L)\right)
$$

of $L / K$ follows via induction on the degree $[L: K]$.

Now, for a finite Galois $p$-extension $L / K$ which is linearly disjoint with $K^{\text {pur }} / K$, where $\operatorname{char}(K)=0$, the $n$-dimensional Hazewinkel map

$$
\mathscr{H}_{L / K}: \mathrm{K}_{n}^{\mathrm{top}}(K) / \mathrm{N}_{L / K}^{\mathrm{top}}\left(\mathrm{K}_{n}^{\mathrm{top}}(L)\right) \rightarrow \operatorname{Gal}(L / K)^{\mathrm{ab}},
$$

of $L / K$ is then defined as the inverse of the $n$-dimensional Neukirch-Iwasawa map

$$
\mathscr{N}_{L / K}^{\mathrm{ab}}: \operatorname{Gal}(L / K)^{\mathrm{ab}} \rightarrow \mathrm{K}_{n}^{\mathrm{top}}(K) / \mathrm{N}_{L / K}^{\mathrm{top}}\left(\mathrm{K}_{n}^{\mathrm{top}}(L)\right)
$$

of $L / K$.

This completes the review of Fesenko's constructive local abelian higher-dimensional class field theory following $[10,11,13]$.

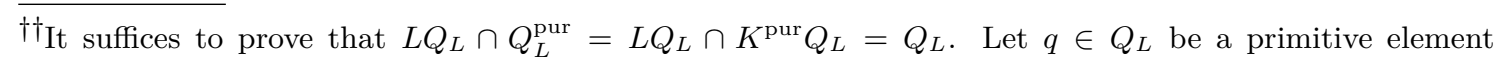
over $K$; namely, let $Q_{L}=K(q)$. Let $B=\left\{1, q, \cdots, q^{s-1}\right\}$ be a basis of the $K$-vector space $Q_{L}$. As $L \cap Q_{L}=K$, the extensions $Q_{L} / K$ and $L / K$ are linearly disjoint. Therefore, $B$ is a basis of the $L$-vector space $L Q_{L}$. Likewise, $B$ is a basis of the $K^{\text {pur }}$-vector space $K^{\text {pur }} Q_{L}=Q_{L}^{\text {pur }}$ since the extension $Q_{L} / K$ is totally ramified. Now, let $a \in L Q_{L} \cap K^{\mathrm{pur}} Q_{L}$. Then there exists unique $\lambda_{0}, \cdots, \lambda_{s-1} \in L$ and there exists unique $\kappa_{0}, \cdots, \kappa_{s-1} \in K^{\text {pur }}$ such that

Therefore,

$$
a=\lambda_{0}+\lambda_{1} q+\cdots+\lambda_{s-1} q^{s-1}=\kappa_{0}+\kappa_{1} q+\cdots+\kappa_{s-1} q^{s-1} .
$$

$$
\left(\lambda_{0}-\kappa_{0}\right)+\left(\lambda_{1}-\kappa_{1}\right) q+\cdots+\left(\lambda_{s-1}-\kappa_{s-1}\right) q^{s-1}=0 .
$$

Now, as $Q_{L} / K$ and $K^{\text {pur }} / K$ are linearly disjoint, it follows that $L^{\text {pur }} \cap Q_{L}^{\text {pur }}=K^{\text {pur }} \Rightarrow L^{\text {pur }} \cap Q_{L}=K$. Thus, $L^{\text {pur }} / K$ and $Q_{L} / K$ are linearly disjoint, which implies that the $K$-basis $B$ of $Q_{L}$ is also an $L^{\text {pur }}$-basis of $\left(L Q_{L}\right)^{\text {pur }}=K^{\text {pur }} L Q_{L}$. Therefore,

$$
\lambda_{0}-\kappa_{0}=\cdots=\lambda_{s-1}-\kappa_{s-1}=0 \Rightarrow \lambda_{0}=\kappa_{0} ; \cdots ; \lambda_{s-1}=\kappa_{s-1} .
$$

The extension $L / K$ is totally ramified. Therefore, $\lambda_{0}, \cdots, \lambda_{s-1} \in K$ and $a=\lambda_{0}+\lambda_{1} q+\cdots+\lambda_{s-1} q^{s-1} \in Q_{L}$, which completes the proof. 


\section{References}

[1] A. Abbes and T. Saito, Ramification of local fields with imperfect residue fields, Amer. J. Math. 124 (5), 879-920, 2002.

[2] E. Artin and J. Tate, Class Field Theory, AMS Chelsea Publishing, Vol.366, American Mathematical Society, Providence, Rhode Island, 2008.

[3] C. Barwick and P. Heine, Pyknotic objects I. Basic notions, arXiv:1904.09966 [math.AG], 2019. Retrieved November 30, 2020, from the arXiv database.

[4] S. Bloch, Algebraic K-theory and class field theory for arithmetic surfaces, Ann. Math. 114, 229-266, 1981.

[5] O. Braunling, M. Groechenig and J. Wolfson, Geometric and analytic structures on higher adèles, Res. Math. Sci. 3, Paper No. 22, 56 pages, 2016.

[6] A. Cámara, Topology on rational points over n-local fields, Rev. R. Acad. Cienc. Exactas Fís. Nat. Ser. A Math. RACSAM 110 (2), 417-432, 2016.

[7] I.B. Fesenko, Class field theory of multi-dimensional local fields of characteristic zero, with residue field of positive characteristic, Algebra i Analiz 3 (3), 165-196, 1991.

[8] _ On class field theory of multi-dimensional local fields of positive characteristic, Algebraic K-theory, Adv. Soviet Math., Vol. 4, Amer. Math. Soc., Providence, RI, 1991, 103-127.

[9] _ Multi-dimensional local class field theory, Dokl. Akad. Nauk SSSR 318 (1), 47-50, 1991.

[10] _ Abelian local p-class field theory, Math. Anal. 301, 561-586, 1995.

[11] _ Abelian extensions of complete discrete valuation fields, Number Theory: Séminaire de Théorie des Nombres de Paris 1993-94 (Sinnou David ed.), Cambridge Univ. Press, Cambridge, 47-74, 1996.

[12] _ Topological Milnor K-groups of higher local fields, Invitation to Higher Local Fields (Ed. I. B. Fesenko, M. Kurihara), Geometry \& Topology Monographs 3, Warwick, 61-74, 2000.

[13] __ Explicit higher local class field theory, Invitation to Higher Local Fields (Ed. I. B. Fesenko, M. Kurihara), Geometry \& Topology Monographs 3, Warwick, 95-101, 2000 .

[14]_, Sequential topologies and quotients of the Milnor K-groups of higher local fields, Algebra i Analiz 13 (3), 198-221, 2001.

[15] I.B. Fesenko and S.V. Vostokov, Local Fields and Their Extensions (2 ${ }^{\text {nd }}$ ed.), AMS Translations of Mathematical Monographs 121, AMS, Providence, Rhode Island, 2002.

[16] H. Hasse, Die Normenresttheorie relative-Abelscher Zahlkörper als Klassenkörper im Kleinen, J. Reine Angew. Math. (Crelle) 162, 145-154, 1930.

[17] M. Hazewinkel, Local class field theory is easy, Advances in Math. 18 (2), 148-181, 1975.

[18] A. Huber, On the Parshin-Beìlinson adèles for schemes, Abh. Math. Sem. Univ. Hamburg 61, 249-273, 1991.

[19] O. Hyodo, Wild ramification in the imperfect residue field case, Galois Groups and Their Representations (Nagoya, 1981), Adv. Stud. Pure Math. 2, North-Holland, Amsterdam, 287-314, 1983.

[20] K.I. Ikeda and E. Serbest, Local non-abelian Kato-Parshin reciprocity law, in preparation.

[21] K. Iwasawa, Local Class Field Theory, Oxford Mathematical Monographs, Oxford Univ. Press., Clarendon, 1986.

[22] K. Kato, A generalization of local class field theory by using K-groups I, II, III, J. Fac. Sci. Univ. Tokyo Sect. IA Math. 26, 303-376, 1979; 27, 603-683, 1980; 29, 31-43, 1982. 
[23] _ Vanishing cycles, ramification of valuations, and class field theory, Duke Math. J. 55 (3), 629-659, 1987.

[24] _ Swan conductors for characters of degree one in the imperfect residue field case, Algebraic K-theory and Algebraic Number Theory (Honolulu, HI, 1987), Contemp. Math. 83, Amer. Math. Soc., Providence, RI, 101-131, 1989.

[25] __ Existence theorem for higher local fields, Invitation to Higher Local Fields (Ed. I. B. Fesenko, M. Kurihara), Geometry \& Topology Monographs 3, Warwick, $165-195,2000$.

[26] K. Kato and S. Saito, Two-dimensional class field theory, Galois Groups and Their Representations (Nagoya, 1981), Adv. Stud. Pure Math. 2, North-Holland, Amsterdam, 103-152, 1983.

[27] _ Global class field theory of arithmetic schemes, Applications of Algebraic $K$-theory to Algebraic Geometry and Number Theory, Part I, II (Boulder, Colorado, 1983), Contemp. Math., 55, Amer. Math. Soc., Providence, RI, 255-331, 1986.

[28] K. Kato and T. Saito Coincidence of two Swan conductors of abelian characters, Épijournal Géom. Algébrique 3, Art. 15, 16 pp, 2019.

[29] Y. Kawada and I. Satake, Class formations II, J. Fac. Sci. Univ. Tokyo 7, 453-490, 1955.

[30] Y. Koya, A generalization of class formation by using hypercohomology, Invent. Math. 101, 705-715, 1990.

[31] _ A generalization of Tate-Nakayama theorem by using hypercohomology, Proc. Japan Acad., Ser. A 69 (3), 53-57, 1993.

[32] _ Class field theory without theorem 90, Algebra Colloq. 1 (4), 347-358, 1994.

[33] K. Kurano and K. Shimomoto, An elementary proof of the Cohen-Gabber theorem in the equal characteristic $p>0$ case, Tohoku Math. J. 70, 377-389, 2018.

[34] S. Lichtenbaum, The construction of weight-two arithmetic cohomology, Invent. Math. 88, 183-215, 1987.

[35] V.G. Lomadze, On the ramification theory of two-dimensional local fields, Math. USSR Sbornik 37, 349-365, 1980.

[36] A.I. Madunts and I.B. Zhukov, Multi-dimensional complete fields : Topology and other basic constructions, Trudy S.-Peterb. Mat. Obshch. 1995, English translation in Amer. Math. Soc. Transl. (Ser. 2)165, 1-34, 1995.

[37] M. Morrow, An introduction to higher dimensional local fields and adèles, arXiv:1204.0586v2 [math.AG], 2012. Retrieved October 25, 2020, from the arXiv database.

[38] _ Continuity of the norm map on Milnor K-theory, J. K-Theory 9 (3), 565$577,2012$.

[39] J. Neukirch, Neubegründung der Klassenkörpertheorie, Math. Z. 186, 557-574, 1984.

[40] _ Class Field Theory, Springer-Verlag, Berlin, 1986.

[41] D.V. Osipov, n-dimensional local fields and adèles on n-dimensional schemes, Surveys in Contemporary Mathematics, London Math. Soc. Lecture Note Ser., 347, Cambridge Univ. Press, Cambridge, 131-164, 2008.

[42] A.N. Parshin, Class fields and algebraic K-theory, Uspekhi Mat. Nauk 30 (1), 253$254,1975$.

[43] Izv. Akad. Nauk SSSR Ser. Mat. 40 (4), 736-773, 1976.

[44] _ Abelian coverings of arithmetic schemes, Sov. Math., Dokl. 19, 1438-1442, 1978.

[45] _ Local class field theory, Trudy Mat. Inst. Steklov 165, 143-170, 1985.

[46] P. Scholze, Lectures on Condensed Mathematics-Joint work with D. Clausen, Bonn Lectures, 2019. 
[47] M. Spiess, Class formations and higher dimensional local class field theory, Journal of Number Theory 62, 273-283, 1997.

[48] J. Tate, The higher dimensional cohomology groups of class field theory, Ann. of Math. (2nd Series) 56, 294-297, 1952.

[49] L. Xiao and I. B. Zhukov, Ramification of higher local fields, approaches and questions, Algebra i Analiz 26 (5), 1-63, 2014.

[50] I.B. Zhukov, Higher dimensional local fields, Invitation to Higher Local Fields (Münster, 1999) (Ed. I. B. Fesenko, M. Kurihara), Geom. Topol. Monogr. 3, Geom. Topol. Publ., Coventry, 5-18, 2000.

[51] _ An approach to higher ramification theory, Invitation to Higher Local Fields (Münster, 1999) (Ed. I. B. Fesenko, M. Kurihara), Geom. Topol. Monogr., 3, Geom. Topol. Publ., Coventry, 143-150, 2000. 